\title{
Environmental pollution and social factors as contributors to preterm birth in Fresno County
}

\author{
Amy M. Padula ${ }^{1 *}$ D, Hongtai Huang ${ }^{1,2}$, Rebecca J. Baer ${ }^{3}$, Laura M. August ${ }^{4}$, Marta M. Jankowska ${ }^{5}$, \\ Laura L. Jellife-Pawlowski ${ }^{6}$, Marina Sirota ${ }^{2}$ and Tracey J. Woodruff ${ }^{1}$
}

\begin{abstract}
Background: Environmental pollution exposure during pregnancy has been identified as a risk factor for preterm birth. Most studies have evaluated exposures individually and in limited study populations.

Methods: We examined the associations between several environmental exposures, both individually and cumulatively, and risk of preterm birth in Fresno County, California. We also evaluated early $(<34$ weeks) and spontaneous preterm birth. We used the Communities Environmental Health Screening Tool and linked hospital discharge records by census tract from 2009 to 2012. The environmental factors included air pollution, drinking water contaminants, pesticides, hazardous waste, traffic exposure and others. Social factors, including area-level socioeconomic status (SES) and race/ ethnicity were also evaluated as potential modifiers of the relationship between pollution and preterm birth.

Results: In our study of 53,843 births, risk of preterm birth was associated with higher exposure to cumulative pollution scores and drinking water contaminants. Risk of preterm birth was twice as likely for those exposed to high versus low levels of pollution. An exposure-response relationship was observed across the quintiles of the pollution burden score. The associations were stronger among early preterm births in areas of low SES.

Conclusions: In Fresno County, we found multiple pollution exposures associated with increased risk for preterm birth, with higher associations among the most disadvantaged. This supports other evidence finding environmental exposures are important risk factors for preterm birth, and furthermore the burden is higher in areas of low SES.

This data supports efforts to reduce the environmental burden on pregnant women.
\end{abstract}

Keywords: Preterm birth, Environmental exposure, Social factors, Prematurity, Pollution

\section{Background}

Preterm birth (before 37 weeks gestation) is estimated to impact $10 \%$ of U.S. births annually with resultant potential for developmental and long-term adverse health consequences [1-4]. The estimated overall cost of preterm birth in the U.S. is approximately $\$ 26.2$ billion per year [5]. Preterm birth is a complex phenotype with no single known mechanism or therapeutic strategy. Causes of preterm birth have remained largely unknown [5] and therefore, in most instances, not amenable to effective interventions or prevention.

\footnotetext{
*Correspondence: Amy.Padula@ucsf.edu

'Department of Obstetrics, Gynecology and Reproductive Sciences, University of California, 550 16th Street, Mail Stop 0132, San Francisco, CA 94143, USA Full list of author information is available at the end of the article
}

Several studies have identified important environmental risk factors for preterm birth including prenatal exposure to air pollution $[6,7]$, contaminated water [812], pesticides [13-15], traffic density (i.e. counts of motor vehicles within a given radius) [16], air toxins [17], and persistent organic pollutants [18]. In general, these studies have been relatively small and usually contaminants have been examined in isolation. Disparities in preterm birth have also been shown to exist by socioeconomic status (SES) wherein those with lower SES experience higher rates of preterm birth and other adverse pregnancy outcomes $[19,20]$.

Studies have also shown that exposures to pollutants differ by race/ethnicity and SES [21]. In previous work, we demonstrated that there are $\mathrm{racial} / \mathrm{ethnic}$ disparities

(c) The Author(s). 2018 Open Access This article is distributed under the terms of the Creative Commons Attribution 4.0 International License (http://creativecommons.org/licenses/by/4.0/), which permits unrestricted use, distribution, and 
in exposure to air pollution during pregnancy [22]. Woodruff et al. found that Hispanic, African American and Asian/Pacific Islander mothers in the U.S. experienced higher mean levels of air pollution and were more than twice as likely to live in the most polluted counties compared with non-Hispanic white mothers after controlling for maternal risk factors, region and educational status [22]. Pregnant women who are exposed to multiple environmental chemicals and multiple psychosocial stressors such as neighborhood SES are at greater risk of adverse birth outcomes [23, 24]. The cumulative impacts and potential interactions between elevated exposures to chemical and psychosocial stressors have been referred to as a form of "double jeopardy" [25]. In other words, not only are such women at increased risk due to more cumulative risk factors, but the combination of risk factors is compounding the risk in a multiplicative rather than additive way. In a previous study, we found interactive effects of air pollution and SES that contribute to risk of preterm birth in the San Joaquin Valley of California [26].

Fresno County, in the San Joaquin Valley of California (CA), is an area of known environmental pollution burden [27] and a high prevalence of preterm birth (12.1\% compared to $9.6 \%$ in CA in 2012). Additionally, Fresno County is characterized by diverse race/ethnicity and SES with a majority of the population being of non-white race and of lower SES, which may impact adverse health effects in conjunction with environmental exposure. Our study examines the association between multiple environmental, medical and social factors and preterm birth in Fresno County, CA from 2009 to 2012. Few studies have addressed how these factors may compound one another to contribute to preterm birth. The interaction of environmental, medical and social stressors may be critical in elucidating disparities in preterm birth. Furthermore, uncovering such compounding effects may focus policy and intervention efforts at reducing pollution burden in the most vulnerable communities.

\section{Methods}

\section{Study population}

Birth outcome and maternal demographic information were collected from a linked hospital discharge birth cohort database maintained by the CA Office of Statewide Health Planning and Development (OSHPD) that includes linked information from the State of CA vital records and hospital discharge records (comorbidities were identified from codes in the form of ICD-9-CM diagnoses). From this linked dataset, the study includes race/ethnicity, infant sex, maternal age at delivery, years of education, participation in the Women, Infants, and Children (WIC) food and nutrition service (a Federally-funded supplemental program), payer for delivery costs (i.e., heath insurance status), place of mother's birth, body mass index (BMI) calculated from maternal height and pre-pregnancy weight, preexisting diabetes (ICD-9 code 250 and 648.0), gestational diabetes (648.8), preexisting hypertension (642.0, 642.1, 642.2, 642.7), gestational hypertension (642.3), preeclampsia/eclampsia $(642.4,642.4,642.6)$, infection (646.5, 646.6, 647), anemia (648.2), mental illness (648.4), reported smoking, reported drug abuse, reported alcohol dependence, trimester when prenatal care began, parity, previous preterm birth, previous cesarean section, inter-pregnancy interval, premature rupture of membranes (658.1), mode of delivery (cesarean or vaginal), birth weight, birth date and gestational age at delivery (best obstetric estimate).

The sample was restricted to live-born singleton births with known birth date, birth weight between three standard deviations of mean by week of gestation [28] and gestational age between 20 and 44 weeks with complete information including census tract or zip code and births between 2009 and 2012 in Fresno County, CA.

Methods and protocols for the study were approved by the Committee for the Protection of Human Subjects within the Health and Human Services Agency of the State of California.

\section{CalEnviroScreen}

We used the California Communities Environmental Health Screening Tool (CalEnviroScreen 2.0, released in 2014) to estimate environmental exposures for each census tract in Fresno County [29]. The CalEnviroScreen was developed by CA's Environmental Protection Agency's (CalEPA) Office of Environmental Health Hazard Assessment to evaluate the cumulative existence of multiple pollutants and stressors in communities [30]. CalEnviroScreen is used to identify communities disproportionately burdened by cumulative impacts and identify disadvantaged communities for allocation of cap and trade funds generated under the Global Warming Solutions Act of 2006 [31]. CalEnviroScreen combines multiple sets of data on pollutants and stressors within a census tract into an overall index, which can be used to screen for places with the highest cumulative burdens (https://oehha.ca.gov/calenviroscreen).

CalEnviroScreen 2.0 consists of 19 environmental and population indicators in total, which are aggregated into a final, relative CalEnviroScreen Score (Table 1, Fig. 1). The CalEnviroScreen Score is made up of two key categories and four components of census tract-level indicators: Pollution Burden - Exposures score and Environmental Effects; and Population Characteristics - Sensitive Populations and Socioeconomic Factors (Fig. 1). Exposures score indicators include measures of pollutant sources, releases and environmental concentrations. Environmental Effects indicators are measures of threats to the environment and degraded ecosystems caused by pollution. In calculating 
Table 1 Description of pollution indicators in CalEnviroScreen 2.0

\begin{tabular}{|c|c|c|c|}
\hline & & Indicators & Description \\
\hline & & Pollution Burden & $\begin{array}{l}\text { Average of percentiles from Exposure and Environmental Effects indicators, } \\
\text { with a half weighting for the Environmental Effects indicators) }\end{array}$ \\
\hline \multirow{24}{*}{$\begin{array}{l}\text { Pollution } \\
\text { Burden }\end{array}$} & \multirow[t]{6}{*}{ Exposures } & Ozone & Amount of daily maximum 8-h Ozone concentration (ppm) \\
\hline & & $\mathrm{PM}_{2.5}$ & Annual mean particulate matter $<2.5 \mu \mathrm{m}$ concentrations $\left(\mu \mathrm{g} / \mathrm{m}^{3}\right)$ \\
\hline & & Diesel PM & Diesel PM emissions from on-road and non-road sources (kg/day) \\
\hline & & Pesticides & $\begin{array}{l}\text { Total pounds of selected active pesticide ingredients (filtered for hazard and volatility) } \\
\text { used in production-agriculture per square mile in the census tract }\end{array}$ \\
\hline & & Toxic Release & $\begin{array}{l}\text { Toxicity-weighted concentrations of modeled chemical releases to air from facility } \\
\text { emissions and off-site incineration }\end{array}$ \\
\hline & & Traffic & $\begin{array}{l}\text { Traffic density, in vehicle-kilometers per hour per road length, within } 150 \mathrm{~m} \text { of the } \\
\text { census tract boundary }\end{array}$ \\
\hline & \multirow{13}{*}{$\begin{array}{l}\text { Individual Drinking } \\
\text { Water Contaminants } \\
\text { of Violation Measures }\end{array}$} & Drinking Water Score & Drinking water contaminant index for selected contaminants \\
\hline & & Arsenic & Arsenic average (ppb) \\
\hline & & Cadmium & Cadmium average (ppb) \\
\hline & & $\mathrm{DBCP}$ & 1,2-Dibromo-3-chloropropane average (ppb) \\
\hline & & Lead & Lead average (ppb) \\
\hline & & Nitrate & Nitrate (as $\mathrm{NO}_{3}$ ) average (ppm) \\
\hline & & Perchlorate & Perchlorate average (ppb) \\
\hline & & TCE & Trichloroethylene average (ppb) \\
\hline & & $\mathrm{TCP}$ & 1,2,3-trichloropropane average (ppb) \\
\hline & & THM & Total trihalomethane average (ppb) \\
\hline & & Uranium & Uranium average $(\mathrm{PCl} / \mathrm{L})$ \\
\hline & & MCL Violations & $\begin{array}{l}\text { The total number of Maximum Contaminant Level (MCL) violations for any chemical } \\
\text { by system from } 2008 \text { to } 2012 \text { population weighted to the census tract }\end{array}$ \\
\hline & & TCR Violations & $\begin{array}{l}\text { Total coliform rule violations by system from } 2008 \text { to } 2012 \text { population weighted } \\
\text { to the census tract }\end{array}$ \\
\hline & \multirow[t]{5}{*}{$\begin{array}{l}\text { Environmental } \\
\text { Effects }\end{array}$} & Groundwater Threats & $\begin{array}{l}\text { Groundwater threats, sum of weighted GeoTracker leaking underground storage tank } \\
\text { sites within buffered distances to populated blocks of census tracts }\end{array}$ \\
\hline & & Hazardous Waste & $\begin{array}{l}\text { Sum of weighted hazardous waste facilities and large quantity generators within buffered } \\
\text { distances to populated blocks of census tracts }\end{array}$ \\
\hline & & Impaired Water Bodies & $\begin{array}{l}\text { Impaired water bodies, sum of number of pollutants across all impaired water bodies } \\
\text { within buffered distances to populated blocks of census tracts }\end{array}$ \\
\hline & & Solid Waste & $\begin{array}{l}\text { Sum of weighted solid waste sites and facilities within buffered distances to populated } \\
\text { blocks of census tracts }\end{array}$ \\
\hline & & Cleanup Sites & $\begin{array}{l}\text { Cleanup sites, sum of weighted EnviroStor cleanup sites within buffered distances } \\
\text { to populated blocks of census tracts }\end{array}$ \\
\hline \multirow{4}{*}{\multicolumn{2}{|c|}{ Socioeconomic Factors }} & Poverty & Percent of population living below two times the federal poverty level \\
\hline & & Unemployment & $\begin{array}{l}\text { Percent of the population over the age of } 16 \text { that is unemployed and eligible } \\
\text { for the labor force }\end{array}$ \\
\hline & & Housing Burden & Percent housing burdened low income households \\
\hline & & Linguistic Isolation & Percent limited English speaking households \\
\hline
\end{tabular}

the average Pollution Burden, the Environmental Effects indicators are weighted by half because CalEPA considers the Exposures score indicators to be more direct measures of exposures to pollution (e.g., air pollution monitoring). These indicators likely contribute more to a person's total pollution burden than the impact of living near contaminated land or water, where the exposure is less immediate. Indicators of Sensitive Populations and Socioeconomic
Factors include both biological traits (e.g., age and health conditions of tract residents) and factors related to tract-level SES (e.g., poverty and education) that can increase susceptibility to the adverse health impacts of pollutants. These together form the Population Characteristics score. The Pollution Burden and Population Characteristics scores are then multiplied together to arrive at a final relative CalEnviroScreen score ranging from 0 to 100 . The 


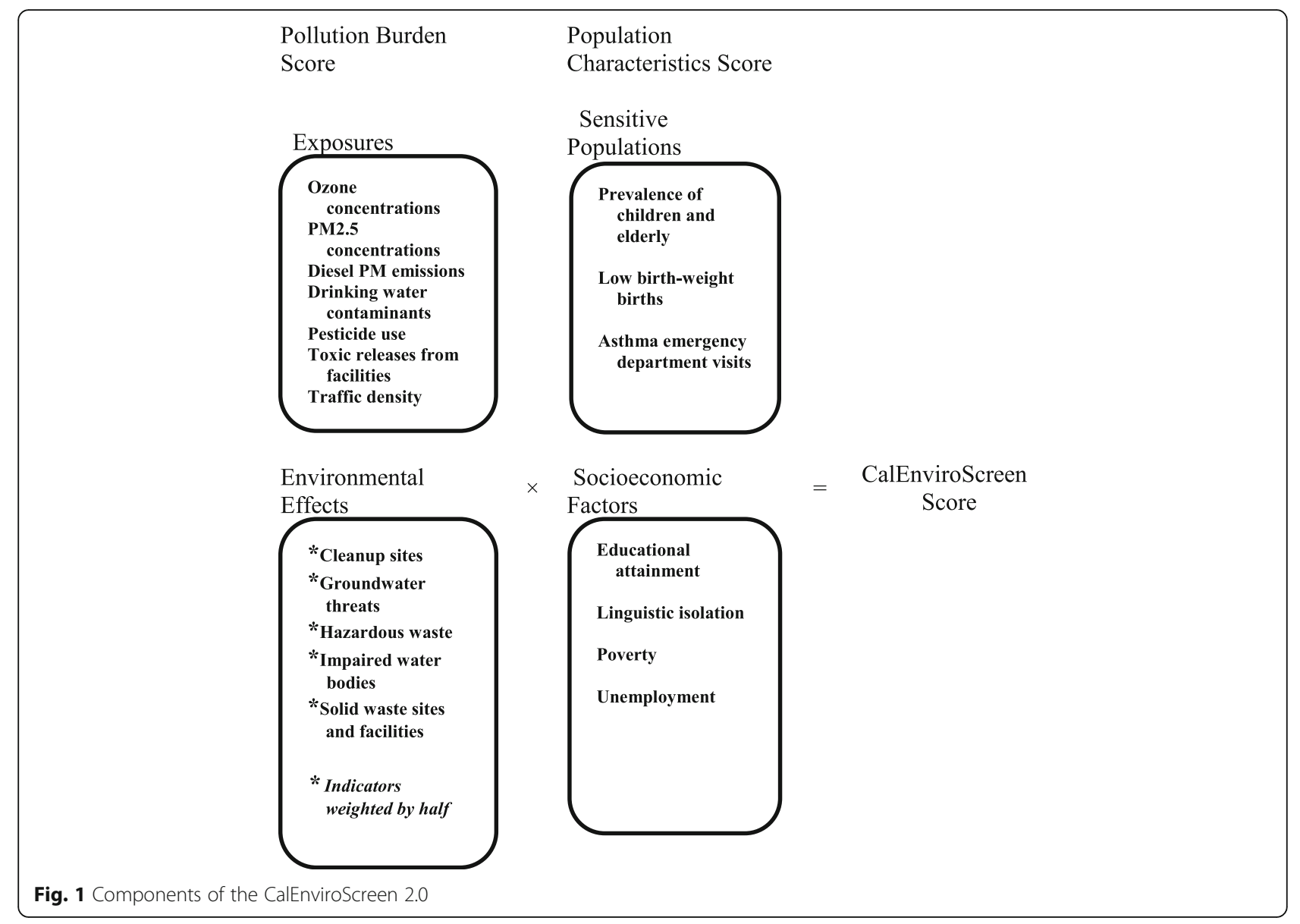

indicators are ranked into percentiles, which allows them to be compared across the state. The indicator percentiles and component scores are also useful to evaluate and understand the key drivers of vulnerability in a community. The methodology and rationale for each specific indicator is described in detail in the CalEnviroScreen 2.0 report [31]. In addition, the individual drinking water contaminants are shown in Table 1. We used the Socioeconomic Factors score from the CalEnviroScreen, which includes the following variables derived from the US Census American Community Survey: educational attainment, linguistic isolation (households where no one over 14 years of age speaks English very well), poverty and unemployment.

We merged the OSHPD birth records with CalEnviroScreen 2.0 data by 2010 census tract. When birth records contained 2000 census tracts, we used the relationship files for 2000 to 2010 census tracts to create area-weighted values for the CalEnviroScreen variables [32]. If a census tract identifier for a birth record was missing or invalid, zip codes were used as surrogate and similar area-weighted adjustments were made using zip code to census tract relationship files $(N=1879 ; 3.5 \%)$.

\section{Statistical analyses}

Our primary outcome was preterm birth was defined as birth at less than 37 weeks gestation. We examined 24 exposure variables, which included the following scores and indicators from the CalEnviroScreen: Pollution Burden Score; Exposures score (component of Pollution Burden); Environmental Effects (component of Pollution Burden); 11 indicators (6 Exposures and 5 Environmental Effects); and 10 subcategories of the drinking water indicator (Fig. 1, Table 1). Each exposure variable was examined separately and classified dichotomously (split at the median) and by quintiles. We calculated Pearson correlation coefficients between the each of the indicators and scores from the CalEnviroScreen.

We examined several sets of covariates and their relationships to preterm birth and exposure indicators, which included socioeconomic variables (maternal education, payer for delivery), demographic characteristics (race/ethnicity, maternal age, maternal country of birth), obstetrical-related variables (diabetes, hypertension, smoking/alcohol/drug use during pregnancy, BMI, parity), and, among multiparous women, previous caesarean section, previous preterm birth, and inter-pregnancy interval from the previous live birth to 
the estimated conception of the index pregnancy. Inter-pregnancy interval was calculated from previous live birth (month and year) as reported in linked records and estimated as months to conception of the index pregnancy. Given that the day of previous live birth was not available, the middle of the month was used for calculation purposes [33]. We explored the association between the covariates and both the outcome (preterm birth) and exposure (above median levels of Pollution Burden).

We used logistic regression to evaluate the association between each indicator and preterm birth ( $<37$ weeks) and early preterm birth (<34 weeks), comparing each of the higher 4 quintiles to the lowest to allow for non-monotonic relationships across the pollution distribution. We ran three sets of models: crude, adjusted with a priori variables and a stepwise selection. The covariates determined a priori included maternal education, age, race/ethnicity, and payer of delivery costs. The stepwise procedure included a forward and backward algorithm to estimate the association between environmental factors with preterm birth that allowed inclusion of covariates listed above that had $p<0.05$ in crude risk calculations.

To explore the hypothesis that there is a double jeopardy when populations are vulnerable to both social and environmental stressors, we examined SES and race/ ethnicity as potential modifiers in the relationship between environmental contaminants and preterm birth. We stratified analyses to examine the relationships between pollution and preterm birth by high and low SES of the census tract the woman lived in. The low SES group consisted of census tracts with below median levels of poverty, education, unemployment and linguistic isolation (Fig. 1, Table 1). We also stratified the analyses by broad race/ethnicity groups: White/ non-Hispanic, non-White/non-Hispanic and Hispanic. These stratified analyses compared above versus below median levels of exposure in Fresno County and risk of preterm birth including early preterm birth.

In sensitivity analyses, we explored several alternative analytic decisions. We evaluated the pollutants continuously, both in individual models and a combined model with social factors. We chose more specific phenotypes of preterm birth including early preterm birth $(<34$ weeks) and spontaneous preterm (i.e. premature labor or premature rupture of membranes) to restrict to preterm births that were not the consequence of a known cause or indication. We evaluated the raw scores of the exposure indicators (as opposed to the percentiles). Additionally, we mapped preterm birth prevalence across the county to visually observe the geographic variability.

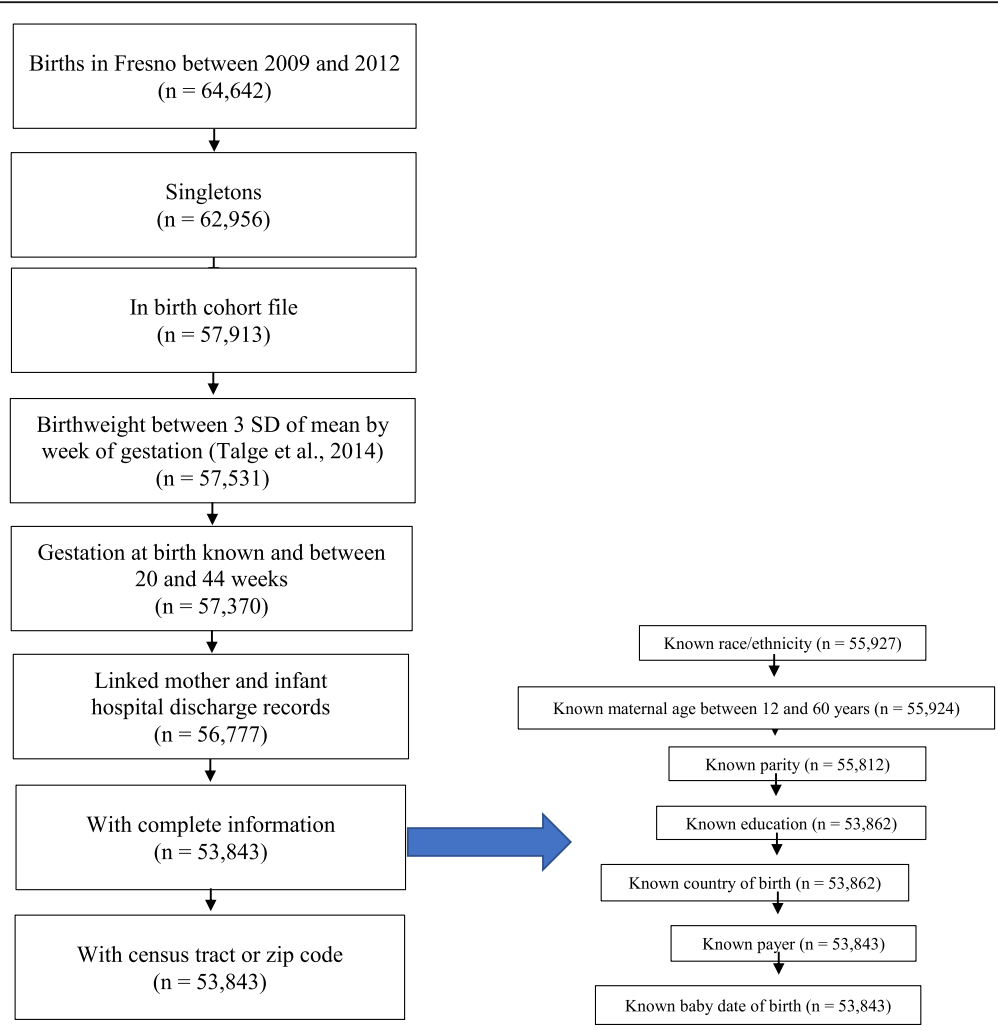

Fig. 2 Flow Chart of Our Study Population of Births in Fresno County, California 
Table 2 Population characteristics in Fresno County, 2009-2012 ( $N=53,843)$

\begin{tabular}{|c|c|c|c|c|c|c|}
\hline \multirow[t]{2}{*}{ Population Characteristics } & \multirow[t]{2}{*}{$n(\%)$} & \multicolumn{5}{|c|}{ Pollution Burden Quintile } \\
\hline & & $1 s t$ & 2nd & $3 r d$ & 4 th & 5 th \\
\hline \multicolumn{7}{|l|}{ Race/ethnicity } \\
\hline White non-Hispanic & $10,620(19.7)$ & $139(35.0)$ & $583(53.5)$ & $2042(38.3)$ & $4113(20.0)$ & $3665(14.0)$ \\
\hline Hispanic & $32,302(60.0)$ & $212(53.4)$ & $357(32.8)$ & $2186(41.0)$ & $12,314(59.7)$ & $17,210(65.5)$ \\
\hline Black & $3095(5.8)$ & $*$ & $17(1.6)$ & $226(4.2)$ & $1159(5.6)$ & $1689(6.4)$ \\
\hline Asian & $5675(10.5)$ & * & $100(9.2)$ & $512(9.6)$ & $2141(10.4)$ & $2899(11.0)$ \\
\hline American Indian/Alaska native & $546(1.0)$ & $19(4.8)$ & * & $70(1.3)$ & $201(1.0)$ & $245(0.9)$ \\
\hline Hawaiian/Pacific Islander & $70(0.1)$ & * & * & * & $40(0.2)$ & $19(0.1)$ \\
\hline Other race & $581(1.1)$ & * & * & $171(3.2)$ & $244(1.2)$ & $155(0.6)$ \\
\hline Two or more races & $954(1.8)$ & * & * & $122(2.3)$ & $406(2.0)$ & $395(1.5)$ \\
\hline \multicolumn{7}{|l|}{ Infant sex } \\
\hline Male & $27,354(50.8)$ & $208(52.4)$ & $569(52.3)$ & $2675(50.1)$ & $10,437(50.6)$ & $13,391(51.0)$ \\
\hline Female & $26,489(49.2)$ & $189(47.6)$ & $520(47.8)$ & $2663(49.9)$ & $10,180(49.4)$ & $12,886(49.0)$ \\
\hline \multicolumn{7}{|l|}{ Maternal age at delivery (years) } \\
\hline$<18$ & $2263(4.2)$ & $19(4.8)$ & $22(2.0)$ & $132(2.5)$ & $801(3.9)$ & $1288(4.9)$ \\
\hline $18-34$ & $45,552(84.6)$ & $340(84.6)$ & $864(79.3)$ & $4420(82.8)$ & $17,506(84.9)$ & $22,331(85.0)$ \\
\hline$>34$ & $6028(11.2)$ & $38(9.6)$ & $203(18.6)$ & $786(14.7)$ & $2311(11.2)$ & $2658(10.1)$ \\
\hline \multicolumn{7}{|l|}{ Maternal education (years) } \\
\hline$<12$ & $16,607(30.8)$ & $85(21.4)$ & $132(12.1)$ & 990 (18.6) & $5877(28.5)$ & $9522(36.2)$ \\
\hline 12 & $15,195(28.2)$ & $159(40.1)$ & $232(21.3)$ & $1275(23.9)$ & $6063(29.4)$ & $7456(28.4)$ \\
\hline$>12$ & $22,041(40.9)$ & $153(38.5)$ & $725(66.6)$ & $3073(57.6)$ & $8678(42.1)$ & $9299(35.4)$ \\
\hline \multicolumn{7}{|l|}{ WIC participant ${ }^{a}$} \\
\hline Yes & $39,404(73.2)$ & $287(72.4)$ & $436(40.0)$ & $2760(51.7)$ & $15,190(73.7)$ & $20,723(79.0)$ \\
\hline No & $14,439(26.8)$ & $150(37.8)$ & $655(60.2)$ & $2532(47.4)$ & $5305(25.7)$ & $5192(9.8)$ \\
\hline \multicolumn{7}{|l|}{ Payer for delivery costs } \\
\hline Private insurance & $13,949(25.9)$ & $150(37.8)$ & $655(60.2)$ & $2532(47.4)$ & $5305(25.7)$ & $5192(9.8)$ \\
\hline Medi-Cal & $39,040(72.5)$ & $222(55.9)$ & $399(36.6)$ & $2730(51.1)$ & $15,015(72.8)$ & $20,665(78.6)$ \\
\hline Other government payer & $651(1.2)$ & * & $22(2.0)$ & $20(0.4)$ & $36(0.2)$ & $43(0.2)$ \\
\hline Self-pay & $134(0.3)$ & * & * & $50(0.9)$ & $230(1.1)$ & $346(1.3)$ \\
\hline Other payer & $2(0.0)$ & * & * & * & $*$ & * \\
\hline No pay & $67(0.1)$ & $*$ & * & * & $31(0.2)$ & $30(0.1)$ \\
\hline \multicolumn{7}{|l|}{ Place of mother's birth } \\
\hline United States & $35,911(66.7)$ & $317(79.9)$ & $825(75.8)$ & $3881(72.7)$ & $13,930(67.6)$ & $16,854(64.1)$ \\
\hline Mexico & $13,174(24.5)$ & $68(17.1)$ & $121(11.1)$ & $737(13.8)$ & $4865(23.6)$ & $7382(28.1)$ \\
\hline Other & $4758(8.8)$ & $12(3.0)$ & $143(13.1)$ & $720(13.5)$ & $1823(8.8)$ & $2041(7.8)$ \\
\hline \multicolumn{7}{|l|}{ Maternal conditions ${ }^{b}$} \\
\hline Diabetes, preexisting & $565(1.1)$ & * & * & $33(0.6)$ & $220(1.7)$ & $302(1.2)$ \\
\hline Diabetes, gestational & $4875(9.1)$ & $42(10.6)$ & $86(7.9)$ & $429(8.0)$ & $1796(8.7)$ & 2517 (9.6) \\
\hline Hypertension, preexisting & $915(1.7)$ & * & * & $103(1.9)$ & $351(1.7)$ & $436(1.7)$ \\
\hline Without preeclampsia & $649(1.2)$ & * & * & $75(1.4)$ & $254(1.2)$ & $298(1.1)$ \\
\hline With preeclampsia & $266(0.5)$ & * & * & $28(0.5)$ & $97(0.5)$ & $138(0.5)$ \\
\hline Hypertension, gestational & $3004(5.6)$ & * & $47(4.3)$ & $294(5.5)$ & $1083(5.3)$ & $1564(6.0)$ \\
\hline Without preeclampsia & $1224(2.3)$ & * & $21(1.9)$ & $140(2.6)$ & $424(2.1)$ & $629(2.4)$ \\
\hline With preeclampsia & $1780(3.3)$ & * & $26(2.4)$ & $154(2.9)$ & $659(3.2)$ & $936(3.6)$ \\
\hline
\end{tabular}


Table 2 Population characteristics in Fresno County, 2009-2012 ( $N=53,843)$ (Continued)

\begin{tabular}{|c|c|c|c|c|c|c|}
\hline \multirow[t]{2}{*}{ Population Characteristics } & \multirow[t]{2}{*}{$n(\%)$} & \multicolumn{5}{|c|}{ Pollution Burden Quintile } \\
\hline & & $1 s t$ & 2nd & $3 r d$ & 4th & 5 th \\
\hline Infection & $7402(13.8)$ & $45(11.3)$ & $102(9.4)$ & $562(10.5)$ & 2805 (13.6) & $3789(14.8)$ \\
\hline Anemia & $4187(7.8)$ & $31(7.8)$ & $73(6.7)$ & $423(7.9)$ & $1887(9.2)$ & $2725(10.4)$ \\
\hline Mental IIIness & $1231(2.3)$ & * & $35(3.2)$ & $155(2.9)$ & $680(3.3)$ & $954(3.6)$ \\
\hline Reported Smoking & $276(0.5)$ & $27(6.8)$ & $89(8.2)$ & $451(8.5)$ & $1664(8.1)$ & $1947(7.4)$ \\
\hline Reported Drug Abuse & $1835(3.4)$ & * & * & $94(1.8)$ & $423(2.1)$ & $697(2.7)$ \\
\hline Reported Alcohol Dependence & $5147(9.6)$ & & & & & \\
\hline \multicolumn{7}{|l|}{ Trimester when prenatal care began } \\
\hline 1 st & $45,632(84.8)$ & $307(77.3)$ & $940(86.3)$ & 4659 (87.3) & $17,575(85.2)$ & $22,032(83.9)$ \\
\hline 2nd & $4846(9.0)$ & $66(16.6)$ & $93(8.5)$ & $341(6.4)$ & $1726(8.4)$ & $2619(10.0)$ \\
\hline $3 r d$ & $696(1.3)$ & * & $20(1.8)$ & $76(1.4)$ & $246(1.2)$ & $340(1.3)$ \\
\hline Multiparous sample & 35,638 & 261 & 708 & 3354 & 13,591 & 17,651 \\
\hline Previous Cesarean-section & 9179 (25.8) & $78(29.9)$ & $208(29.4)$ & $886(26.4)$ & $3520(25.9)$ & $4462(25.3)$ \\
\hline Previous Preterm Birth & $403(1.1)$ & * & * & $48(1.4)$ & $168(1.2)$ & $178(1.0)$ \\
\hline \multicolumn{7}{|l|}{ Interpregnancy Interval c } \\
\hline$<6$ months & $2283(6.4)$ & $21(8.1)$ & $28(4.0)$ & $174(5.2)$ & $853(6.3)$ & $1207(6.8)$ \\
\hline 6-23 months & $11,683(32.8)$ & $76(29.1)$ & $271(38.3)$ & $1142(34.1)$ & $4407(32.4)$ & $5748(32.6)$ \\
\hline 24-59 months & $13,671(38.4)$ & $112(42.9)$ & $253(35.7)$ & $1233(36.8)$ & $5208(38.3)$ & $6843(38.8)$ \\
\hline$>59$ months & $5371(15.1)$ & $34(13.0)$ & $95(13.4)$ & $527(15.7)$ & 2099 (15.4) & $2610(14.8)$ \\
\hline
\end{tabular}

${ }^{*} n<16$

${ }^{a}$ WIC Participation - Women, Infants and Children food and nutrition service

betermined by ICD-9 codes in maternal discharge records: preexisting diabetes (ICD-9 code 250 and 648.0), gestational diabetes (648.8), preexisting hypertension (642.0,

642.1, 642.2, 642.7), gestational hypertension (642.3), preeclampsia/eclampsia (642.4, 642.4, 642.6), infection (646.5, 646.6, 647), anemia (648.2), mental illness (648.4)

cNumber of months between the delivery date of the preceding live birth and the conception date of the index pregnancy

\section{Results}

\section{Population characteristics}

After applying our exclusion criteria, our final study population included 53,843 births (Fig. 2). Our study population was highly diverse in both race/ethnicity and SES and pollution burden was higher in non-White and low SES areas (Table 2). We did not present cells with less than 16 women (for privacy purposes) nor calculate odds ratios with any cell less than 5 . Our population in Fresno County was majority Hispanic (60\%), followed by non-Hispanic white (19.7\%), Asian (10.5\%), and African American (5.8\%). One quarter of mothers were born in Mexico (24.5\%). More than $30 \%$ of the mothers had less than high school education and more than two-thirds of the mothers' delivery costs were paid by Medi-Cal (California's Medicaid). The prevalence of preterm birth ( $<37$ weeks), early preterm birth $(<34$ weeks) and spontaneous preterm birth $(<37$ weeks and premature rupture of membranes or preterm labor) were $8.5 \%, 2.1 \%$ and $7 \%$, respectively.

Correlations were moderate between diesel PM, ozone and traffic, ranging from 0.53 to 0.79 (Additional file 1: Appendix 1a). Nitrate and TCE were also moderately correlated (0.62; Additional file 1: Appendix 1b). Summary statistics of each of the indicators by preterm birth status is presented in Table 3. Although many are similar between the two groups, the Exposures score, $\mathrm{PM}_{2.5}$, Diesel PM, Toxic Release, Traffic, Drinking Water Score, Cadmium, Nitrate, Uranium, Solid Waste and Pollution Burden Score were all higher among preterm births.

Associations between the covariates and preterm birth included hypertension with pre-eclampsia, drug or alcohol abuse and previous preterm birth as maternal factors strongly associated with preterm birth (data not shown). Additionally, Hispanic, African-American and Asian mothers were more likely to have preterm birth compared to white mothers. Mothers with Medi-Cal payer status had higher risk of preterm birth. Additional risk factors for preterm birth included underweight BMI, diabetes, hypertension without pre-eclampsia, infection, anemia, mental illness, previous cesarean delivery, and short (<6 months) or long (> 59 months) inter-pregnancy interval. Conversely, mothers that participated in WIC were less likely to deliver preterm.

\section{Association between environmental pollutants and preterm birth}

We found that the mothers in the highest quintile of Exposures score were two times as likely to have preterm 
Table 3 Descriptive statistics of environmental indicators by preterm birth status in Fresno County, 2009-2012 ( $N=53,843)$

\begin{tabular}{lll}
\hline $\begin{array}{l}\text { Environmental } \\
\text { exposure }\end{array}$ & Preterm Birth & Full Term Birth \\
& $<37$ weeks $(N=4560)$ & $\geq 37$ weeks $(N=49,283)$ \\
\hline
\end{tabular}

Exposures Score

$\begin{array}{lll}\text { Mean (SD) } & 64.46(9.83) & 63.53(10.34) \\ \text { Median (IQR) } & 65.23(60.15-70.14) & 64.71(58.94-69.47)\end{array}$

Ozone

$\begin{array}{lll}\text { Mean }(\mathrm{SD}) & 0.31(0.09) & 0.31(0.09) \\ \text { Median (IQR) } & 0.32(0.27-0.37) & 0.32(0.28-0.38)\end{array}$

Pesticides

$\begin{array}{lll}\text { Mean (SD) } & 452.78(912.72) & 477.23(965.8) \\ \text { Median (IQR) } & 10.33(0.00-505.73) & 10.33(0.00-524.69)\end{array}$

$\mathrm{PM}_{2.5}$

Mean (SD) $14.18(1.13)$

Median (IQR) 14.28 (13.89-14.53)

Diesel PM

$\begin{array}{lll}\text { Mean (SD) } & 25.99(17.66) & 24.92(17.54) \\ \text { Median (IQR) } & 22.96(7.93-42.94) & 20.79(7.38-41.76)\end{array}$

Toxic Release

Mean (SD) $\quad 3111.81(9510.54) \quad 2874.46$ (9198.78)

Median (IQR) 469.15 (272.01-1109.65) 381.64 (236.93-1037.49) Traffic

Mean (SD) $692.44(471.82)$

Median (IQR) $621.44(299.40-941.13)$

Drinking Water

Mean (SD) 454.91 (114.09)

Median (IQR) 406.83 (406.83-513.41)

Arsenic

Mean (SD) $1.38(2.26)$

Median (IQR) $0.70(0.70-0.84)$

Cadmium

Mean (SD) $\quad 0.0007(0.0076)$

Median (IQR) $0.00(0.00-0.00)$

1,2-Dibromo-3-chloropropane (DBCP)

Mean (SD) $\quad 0.03(0.02)$

Median (IQR) $0.03(0.03-0.03)$

Hexavalent chromium

$\begin{array}{ll}\text { Mean (SD) } & 0.27(0.63) \\ \text { Median (IQR) } & 0.00(0.00-0.06)\end{array}$

Lead

$$
\begin{array}{ll}
\text { Mean (SD) } & 0.13(0.40) \\
\text { Median (IQR) } & 0.00(0.00-0.02)
\end{array}
$$

Nitrate

Mean (SD) $21.36(7.36)$

Median (IQR) 25.30 (16.74-25.30)
670.75 (467.61)

$605.60(267.04-929.61)$

453.59 (117.42)

$1.40(2.24)$

$0.70(0.70-0.86)$

$0.0006(0.0068)$

$0.00(0.00-0.00)$

$0.03(0.02)$

$0.03(0.03-0.03)$

$0.27(0.62)$

$0.00(0.00-0.07)$

$0.14(0.43)$

$0.00(0.00-0.02)$

$21.30(7.68)$

$25.30(16.71-25.30)$
$406.83(406.83-514.09)$
Table 3 Descriptive statistics of environmental indicators by preterm birth status in Fresno County, 2009-2012 ( $N=53,843)$

\begin{tabular}{|c|c|c|}
\hline \multirow{2}{*}{$\begin{array}{l}\text { Environmental } \\
\text { exposure }\end{array}$} & Preterm Birth & Full Term Birth \\
\hline & $<37$ weeks $(N=4560)$ & $\geq 37$ weeks $(N=49,283)$ \\
\hline \multicolumn{3}{|l|}{ Perchlorate } \\
\hline Mean (SD) & $0.06(0.33)$ & $0.06(0.32)$ \\
\hline Median (IQR) & $0.00(0.00-0.00)$ & $0.00(0.00-0.00)$ \\
\hline \multicolumn{3}{|c|}{ Trichloroethylene (TCE) } \\
\hline Mean (SD) & $0.10(0.07)$ & $0.09(0.07)$ \\
\hline Median (IQR) & $0.15(0.00-0.15)$ & $0.15(0.00-0.15)$ \\
\hline \multicolumn{3}{|c|}{ Trihalomethane (THM) } \\
\hline Mean (SD) & $4.53(9.80)$ & $5.13(11.22)$ \\
\hline Median (IQR) & $2.66(0.96-2.66)$ & $2.66(0.96-2.66)$ \\
\hline \multicolumn{3}{|l|}{ Uranium } \\
\hline Mean (SD) & $3.38(1.75)$ & $3.36(1.88)$ \\
\hline Median (IQR) & $3.12(3.12-3.18)$ & $3.12(3.12-3.17)$ \\
\hline \multicolumn{3}{|c|}{ Maximum Contaminant Level (MCL) Violations } \\
\hline Mean (SD) & $0.85(1.47)$ & $0.85(1.48)$ \\
\hline Median (IQR) & $1.00(0.00-1.00)$ & $0.99(0.00-1.00)$ \\
\hline \multicolumn{3}{|c|}{ Total coliform rule (TCR) Violations } \\
\hline Mean (SD) & $0.11(0.31)$ & $0.11(0.32)$ \\
\hline Median (IQR) & $0.00(0.00-0.00)$ & $0.00(0.00-0.00)$ \\
\hline \multicolumn{3}{|c|}{ Environmental Effects Score } \\
\hline Mean (SD) & $24.68(19.50)$ & $24.77(19.33)$ \\
\hline Median (IQR) & $20.15(8.30-38.25)$ & $20.45(8.30-38.25)$ \\
\hline \multicolumn{3}{|l|}{ Cleanup Sites } \\
\hline Mean (SD) & $6.21(13.05)$ & $6.32(13.00)$ \\
\hline Median (IQR) & $1.00(0.00-8.00)$ & $1.15(0.00-8.00)$ \\
\hline \multicolumn{3}{|l|}{ Groundwater Threats } \\
\hline Mean (SD) & $15.23(18.67)$ & $15.34(18.78)$ \\
\hline Median (IQR) & $9.56(1.50-20.94)$ & $9.56(1.50-21.00)$ \\
\hline \multicolumn{3}{|l|}{ Hazardous Waste } \\
\hline Mean (SD) & $0.36(1.07)$ & $0.36(1.10)$ \\
\hline Median (IQR) & $0.05(0.00-0.21)$ & $0.05(0.00-0.21)$ \\
\hline \multicolumn{3}{|c|}{ Imperial Water Bodies } \\
\hline Mean (SD) & $0.47(1.35)$ & $0.51(1.36)$ \\
\hline Median (IQR) & $0.00(0.00-0.00)$ & $0.00(0.00-0.00)$ \\
\hline \multicolumn{3}{|l|}{ Solid Waste } \\
\hline Mean (SD) & $1.45(2.76)$ & $1.40(2.76)$ \\
\hline Median (IQR) & $0.00(0.00-2.00)$ & $0.00(0.00-2.00)$ \\
\hline \multicolumn{3}{|l|}{ Pollution Burden Score } \\
\hline Mean (SD) & $6.51(1.04)$ & $6.43(1.07)$ \\
\hline Median (IQR) & $6.33(5.83-7.12)$ & $6.24(5.79-7.09)$ \\
\hline
\end{tabular}
(Continued) 
Table 4 Crude and adjusted odds ratio of preterm birth across quintiles of CalEnviroScreen indicators and scores in Fresno County, 2009-2012 ( $N=53,843)$

\begin{tabular}{|c|c|c|c|c|}
\hline Type of Preterm Birth & $<37$ weeks $(N=4560)$ & $\geq 37$ weeks $(N=49,283)$ & & \\
\hline Environmental exposure & $N(\%)$ & $N(\%)$ & COR $(95 \% \mathrm{Cl})$ & $\mathrm{aOR}^{\mathrm{a}}(95 \% \mathrm{Cl})$ \\
\hline \multicolumn{5}{|l|}{ Exposures Score } \\
\hline 0 - 19th percentile & $17(0.4)$ & $380(0.8)$ & Reference & Reference \\
\hline 20 - 39th percentile & $74(1.6)$ & $863(1.8)$ & $1.84(1.09,3.12)$ & $1.73(1.01,2.97)$ \\
\hline 40 - 59th percentile & $151(3.3)$ & $1834(3.7)$ & $1.78(1.08,2.93)$ & $1.85(1.12,3.06)$ \\
\hline 60 - 79th percentile & $673(14.8)$ & 8666 (17.6) & $1.68(1.04,2.72)$ & $1.64(1.01,2.65)$ \\
\hline 80 - 100th percentile & $3633(79.7)$ & $37,428(76.0)$ & $2.07(1.28,3.33)$ & $2.00(1.25,3.23)$ \\
\hline \multicolumn{5}{|l|}{ Ozone } \\
\hline 0 - 19th percentile & $\dagger$ & $\dagger$ & Reference & Reference \\
\hline 20 - 39th percentile & $\dagger$ & $66(0.1)$ & NC & NC \\
\hline 40 - 59th percentile & $178(3.9)$ & $2100(4.3)$ & NC & NC \\
\hline 60 - 79th percentile & $631(13.8)$ & $6659(13.5)$ & NC & NC \\
\hline 80 - 100th percentile & $3703(81.2)$ & $39,729(80.6)$ & NC & NC \\
\hline \multicolumn{5}{|l|}{ Pesticides } \\
\hline 0 - 19th percentile & $1768(38.8)$ & $19,587(39.7)$ & Reference & Reference \\
\hline 20 - 39th percentile & $357(7.8)$ & $3486(7.1)$ & $1.12(1.00,1.26)$ & $1.13(1.01,1.26)$ \\
\hline 40 - 59th percentile & $284(6.2)$ & $3082(6.3)$ & $1.02(0.90,1.16)$ & $1.00(0.88,1.14)$ \\
\hline 60 - 79th percentile & $723(15.9)$ & 7565 (15.4) & $1.05(0.97,1.15)$ & $1.05(0.96,1.15)$ \\
\hline 80 - 100th percentile & $1428(31.3)$ & $15,563(31.6)$ & $1.02(0.95,1.09)$ & $0.98(0.92,1.06)$ \\
\hline \multicolumn{5}{|l|}{$\mathrm{PM}_{2.5}$} \\
\hline 0 - 19th percentile & $20(0.4)$ & $315(0.6)$ & Reference & Reference \\
\hline 20 - 39th percentile & $\dagger$ & $\dagger$ & $4.19(0.56,31.20)$ & $4.02(0.53,30.23)$ \\
\hline 40 - 59th percentile & $38(0.8)$ & $650(1.3)$ & $0.93(0.54,1.59)$ & $0.89(0.51,1.56)$ \\
\hline 60 - 79th percentile & $37(0.8)$ & $562(1.1)$ & $1.03(0.60,1.78)$ & $1.07(0.59,1.94)$ \\
\hline 80 - 100th percentile & $4295(94.2)$ & 45,804 (92.9) & $1.44(0.93,2.23)$ & $1.36(0.88,2.11)$ \\
\hline \multicolumn{5}{|l|}{ Diesel Particulate Matter } \\
\hline 0 - 19th percentile & $701(15.4)$ & $8391(17.0)$ & Reference & Reference \\
\hline 20 - 39th percentile & $609(13.4)$ & $6616(13.4)$ & $1.09(0.98,1.22)$ & $1.13(1.02,1.27)$ \\
\hline 40 - 59th percentile & $566(12.4)$ & $6582(13.4)$ & $1.03(0.92,1.15)$ & $1.11(0.99,1.25)$ \\
\hline 60 - 79th percentile & $738(16.2)$ & 7690 (15.6) & $1.14(1.02,1.26)$ & $1.20(1.08,1.33)$ \\
\hline 80 - 100th percentile & $1946(52.7)$ & $20,004(40.6)$ & $1.15(1.05,1.25)$ & $1.16(1.06,1.26)$ \\
\hline \multicolumn{5}{|l|}{ Toxic Release } \\
\hline 0 - 19th percentile & $166(3.6)$ & $1915(3.9)$ & Reference & Reference \\
\hline 20 - 39th percentile & $466(10.2)$ & $6073(12.3)$ & $0.89(0.75,1.07)$ & $0.99(0.82,1.19)$ \\
\hline 40 - 59th percentile & $2256(49.5)$ & $25,163(51.1)$ & $1.03(0.88,1.21)$ & $1.10(0.94,1.28)$ \\
\hline 60 - 79th percentile & $1049(23.0)$ & $10,059(20.4)$ & $1.18(1.01,1.39)$ & $1.21(1.03,1.42)$ \\
\hline 80 - 100th percentile & $623(13.7)$ & $6073(12.3)$ & $1.17(0.98,1.38)$ & $1.16(0.97,1.37)$ \\
\hline \multicolumn{5}{|l|}{ Traffic } \\
\hline 0 - 19th percentile & 1668 (36.6) & $18,996(38.5)$ & Reference & Reference \\
\hline 20 - 39th percentile & $962(21.1)$ & $10,572(21.5)$ & $1.03(0.95,1.12)$ & $1.04(0.96,1.12)$ \\
\hline 40 - 59th percentile & $982(21.5)$ & $10,003(20.3)$ & $1.11(1.02,1.20)$ & $1.09(1.01,1.18)$ \\
\hline 60 - 79th percentile & $923(20.2)$ & $9423(19.1)$ & $1.11(1.02,1.20)$ & $1.09(1.00,1.18)$ \\
\hline 80 - 100th percentile & $25(0.6)$ & $289(0.6)$ & $0.99(0.66,1.46)$ & $0.99(0.67,1.47)$ \\
\hline
\end{tabular}


Table 4 Crude and adjusted odds ratio of preterm birth across quintiles of CalEnviroScreen indicators and scores in Fresno County, 2009-2012 ( $N=53,843)$ (Continued)

\begin{tabular}{|c|c|c|c|c|}
\hline Type of Preterm Birth & $<37$ weeks $(N=4560)$ & $\geq 37$ weeks $(N=49,283)$ & & \\
\hline Environmental exposure & $N(\%)$ & $N(\%)$ & cOR (95\% Cl) & $\mathrm{aOR}^{\mathrm{a}}(95 \% \mathrm{Cl})$ \\
\hline \multicolumn{5}{|l|}{ Drinking Water } \\
\hline 0 - 19th percentile & $29(0.6)$ & $527(1.1)$ & Reference & Reference \\
\hline 20 - 39th percentile & t & + & NC & NC \\
\hline 40 - 59th percentile & $382(8.4)$ & $4570(9.3)$ & $1.48(1.01,2.16)$ & $1.50(1.02,2.19)$ \\
\hline 60 - 79th percentile & $2800(61.4)$ & $29,397(59.7)$ & $1.67(1.16,2.40)$ & $1.67(1.16,2.41)$ \\
\hline 80 - 100th percentile & $1337(29.3)$ & $14,677(29.8)$ & $1.60(1.11,2.31)$ & $1.67(1.15,2.41)$ \\
\hline \multicolumn{5}{|l|}{ Arsenic } \\
\hline 0 - 19th percentile & $35(0.8)$ & $397(0.8)$ & Reference & Reference \\
\hline 20 - 39th percentile & $190(4.2)$ & $2323(4.7)$ & $0.93(0.65,1.34)$ & $0.91(0.63,1.31)$ \\
\hline 40 - 59th percentile & $3288(72.1)$ & $34,615(70.2)$ & $1.07(0.77,1.49)$ & $1.04(0.74,1.45)$ \\
\hline 60 - 79th percentile & $455(10.0)$ & $5526(11.2)$ & $0.94(0.67,1.32)$ & $0.93(0.66,1.31)$ \\
\hline 80 - 100th percentile & $580(12.7)$ & $6310(12.8)$ & $1.04(0.74,1.46)$ & $0.98(0.69,1.38)$ \\
\hline \multicolumn{5}{|l|}{ Cadmium } \\
\hline 0 - 19th percentile & $4336(95.1)$ & $46,850(95.1)$ & Reference & Reference \\
\hline 20 - 39th percentile & + & $89(0.2)$ & $0.86(0.41,1.81)$ & $0.86(0.41,1.82)$ \\
\hline 40 - 59th percentile & + & + & NC & NC \\
\hline 60 - 79th percentile & 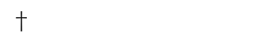 & $\dagger$ & $0.79(0.11,5.59)$ & $0.69(0.10,4.90)$ \\
\hline 80 - 100th percentile & $216(4.7)$ & $2330(4.7)$ & $1.00(0.87,1.15)$ & $1.00(0.87,1.14)$ \\
\hline \multicolumn{5}{|c|}{ 1,2-Dibromo-3-chloropropane (DBCP) } \\
\hline 0 - 19th percentile & + & $\dagger$ & Reference & Reference \\
\hline 20 - 39th percentile & $\dagger$ & $\dagger$ & NC & NC \\
\hline 40 - 59th percentile & $\dagger$ & $\dagger$ & NC & NC \\
\hline 60 - 79th percentile & $216(4.7)$ & $2314(4.7)$ & NC & NC \\
\hline 80 - 100th percentile & $4303(94.4)$ & $46,330(94.0)$ & NC & NC \\
\hline \multicolumn{5}{|l|}{ Hexavalent chromium } \\
\hline 0 - 19th percentile & $2731(59.9)$ & $28,808(58.5)$ & Reference & Reference \\
\hline 20 - 39th percentile & $162(3.6)$ & $1799(3.7)$ & $0.95(0.81,1.12)$ & $0.99(0.85,1.16)$ \\
\hline 40 - 59th percentile & $671(14.7)$ & $7035(14.3)$ & $1.01(0.92,1.09)$ & $1.00(0.92,1.09)$ \\
\hline 60 - 79th percentile & $426(9.3)$ & $5077(10.3)$ & $0.89(0.81,0.99)$ & $0.89(0.81,0.99)$ \\
\hline 80 - 100th percentile & $219(4.8)$ & $2151(4.4)$ & $1.07(0.92,1.22)$ & $1.06(0.92,1.21)$ \\
\hline \multicolumn{5}{|l|}{ Lead } \\
\hline 0 - 19th percentile & $2781(61.0)$ & $29,538(59.9)$ & Reference & Reference \\
\hline 20 - 39th percentile & $51(1.1)$ & $544(1.1)$ & $1.00(0.76,1.31)$ & $1.02(0.77,1.34)$ \\
\hline 40 - 59th percentile & $71(1.6)$ & $869(1.8)$ & $0.88(0.69,1.11)$ & $0.85(0.67,1.07)$ \\
\hline 60 - 79th percentile & $772(16.9)$ & $8534(17.3)$ & $0.96(0.89,1.04)$ & $0.98(0.90,1.06)$ \\
\hline 80 - 100th percentile & $873(19.1)$ & $9686(19.7)$ & $0.96(0.89,1.04)$ & $1.00(0.93,1.08)$ \\
\hline \multicolumn{5}{|l|}{ Nitrate } \\
\hline 0 - 19th percentile & $123(2.7)$ & $1232(2.5)$ & Reference & Reference \\
\hline 20 - 39th percentile & $62(1.4)$ & 807 (1.6) & $0.79(0.58,1.07)$ & $0.77(0.56,1.06)$ \\
\hline 40 - 59th percentile & $56(1.2)$ & $969(2.0)$ & $0.60(0.44,0.83)$ & $0.59(0.42,0.81)$ \\
\hline 60 - 79th percentile & $250(5.5)$ & $2570(5.2)$ & $0.98(0.79,1.21)$ & $1.01(0.81,1.27)$ \\
\hline 80 - 100th percentile & $4057(89.0)$ & $43,593(88.5)$ & $0.94(0.78,1.12)$ & $1.05(0.88,1.26)$ \\
\hline
\end{tabular}


Table 4 Crude and adjusted odds ratio of preterm birth across quintiles of CalEnviroScreen indicators and scores in Fresno County, 2009-2012 ( $N=53,843)$ (Continued)

\begin{tabular}{|c|c|c|c|c|}
\hline Type of Preterm Birth & $<37$ weeks $(N=4560)$ & $\geq 37$ weeks $(N=49,283)$ & & \\
\hline Environmental exposure & $N(\%)$ & $N(\%)$ & cOR (95\% Cl) & $\mathrm{aOR}^{\mathrm{a}}(95 \% \mathrm{Cl})$ \\
\hline \multicolumn{5}{|l|}{ Perchlorate } \\
\hline 0 - 19th percentile & $3928(86.1)$ & $42,060(85.3)$ & Reference & Reference \\
\hline 20 - 39th percentile & $23(0.5)$ & $305(0.6)$ & $0.82(0.54,1.24)$ & $0.88(0.58,1.33)$ \\
\hline 40 - 59th percentile & $90(2.0)$ & $1001(2.0)$ & $0.97(0.78,1.19)$ & $0.99(0.81,1.23)$ \\
\hline 60 - 79th percentile & 164 (3.6) & $1902(3.9)$ & $0.93(0.80,1.09)$ & $0.97(0.83,1.14)$ \\
\hline 80 - 100th percentile & $355(7.8)$ & $4015(8.2)$ & $0.95(0.85,1.06)$ & $0.97(0.87,1.08)$ \\
\hline \multicolumn{5}{|l|}{ Trichloroethylene (TCE) } \\
\hline 0 - 19th percentile & $1244(27.3)$ & $13,123(28.7)$ & Reference & Reference \\
\hline 20 - 39th percentile & $\dagger$ & $100(0.2)$ & $0.24(0.06,0.97)$ & $0.27(0.07,1.07)$ \\
\hline 40 - 59th percentile & $24(0.5)$ & $388(0.8)$ & $0.72(0.48,1.08)$ & $0.76(0.51,1.14)$ \\
\hline 60 - 79th percentile & $1144(25.1)$ & $12,506(25.4)$ & $1.04(0.96,1.12)$ & $1.07(0.98,1.16)$ \\
\hline 80 - 100th percentile & $2134(46.8)$ & $22,054(44.8)$ & $1.09(1.02,1.17)$ & $1.09(1.01,1.17)$ \\
\hline \multicolumn{5}{|l|}{ Trihalomethane (THM) } \\
\hline 0 - 19th percentile & $4041(88.6)$ & $42,863(87.0)$ & Reference & Reference \\
\hline 20 - 39th percentile & $182(4.0)$ & $2235(4.5)$ & $0.87(0.75,1.01)$ & $0.86(0.75,1.00)$ \\
\hline 40 - 59th percentile & $270(5.9)$ & $3131(6.4)$ & $0.92(0.81,1.04)$ & $1.01(0.89,1.15)$ \\
\hline 60 - 79th percentile & $\dagger$ & $112(0.2)$ & $1.12(0.64,1.98)$ & $1.37(0.78,2.43)$ \\
\hline 80 - 100th percentile & $55(1.2)$ & $937(1.9)$ & $0.64(0.49,0.84)$ & $0.62(0.47,0.81)$ \\
\hline \multicolumn{5}{|l|}{ Uranium } \\
\hline 0 - 19th percentile & $29(0.6)$ & $527(1.1)$ & Reference & Reference \\
\hline 20 - 39th percentile & $310(6.8)$ & $3787(7.7)$ & $1.45(0.99,2.12)$ & $1.44(0.98,2.11)$ \\
\hline 40 - 59th percentile & $118(2.6)$ & $1614(3.3)$ & $1.31(0.87,1.96)$ & $1.27(0.85,1.92)$ \\
\hline 60 - 79th percentile & $291(6.4)$ & $3061(6.2)$ & $1.66(1.14,2.44)$ & $1.73(1.18,2.55)$ \\
\hline 80 - 100th percentile & $3668(80.4)$ & $38,646(78.4)$ & $1.66(1.15,2.40)$ & $1.68(1.17,2.43)$ \\
\hline \multicolumn{5}{|c|}{ Maximum Contaminant Level (MCL)Violations } \\
\hline 0 - 19th percentile & $1028(22.5)$ & $11,257(22.8)$ & Reference & Reference \\
\hline 20 - 39th percentile & $\dagger$ & $65(0.1)$ & $0.18(0.03,1.29)$ & $1.20(0.03,1.41)$ \\
\hline 40 - 59th percentile & t & $93(0.2)$ & $0.37(0.12,1.16)$ & $0.40(0.13,1.25)$ \\
\hline 60 - 79th percentile & $43(0.9)$ & $634(1.3)$ & $0.76(0.56,1.03)$ & $0.74(0.54,1.00)$ \\
\hline 80 - 100th percentile & $3473(76.2)$ & $37,122(75.3)$ & $1.02(0.95,1.10)$ & $1.01(0.94,1.09)$ \\
\hline \multicolumn{5}{|c|}{ Total coliform rule (TCR) Violations } \\
\hline 0 - 19th percentile & $3118(68.4)$ & $33,671(68.3)$ & Reference & Reference \\
\hline 20 - 39th percentile & $113(2.5)$ & $1250(2.5)$ & $0.98(0.81,1.18)$ & $1.00(0.83,1.21)$ \\
\hline 40 - 59th percentile & $95(2.1)$ & $953(1.9)$ & $1.07(0.87,1.31)$ & $1.06(0.86,1.30)$ \\
\hline 60 - 79th percentile & $106(2.3)$ & $1267(2.6)$ & $0.91(0.75,1.11)$ & $0.92(0.76,1.13)$ \\
\hline 80 - 100th percentile & $1128(24.7)$ & $12,142(24.6)$ & $1.00(0.94,1.07)$ & $1.00(0.93,1.07)$ \\
\hline \multicolumn{5}{|l|}{ Environmental Effects Score } \\
\hline 0 - 19th percentile & $1725(37.8)$ & $18,282(37.1)$ & Reference & Reference \\
\hline 20 - 39th percentile & $946(20.8)$ & $10,256(20.8)$ & $0.98(0.90,1.06)$ & $0.97(0.90,1.06)$ \\
\hline 40 - 59th percentile & $556(12.2)$ & $6282(12.8)$ & $0.94(0.86,1.04)$ & $0.92(0.84,1.01)$ \\
\hline 60 - 79th percentile & $837(18.4)$ & $9273(18.8)$ & $0.96(0.88,1.04)$ & $0.93(0.85,1.01)$ \\
\hline 80 - 100th percentile & $496(10.9)$ & $5190(10.5)$ & $1.01(0.92,1.12)$ & $0.94(0.85,1.05)$ \\
\hline
\end{tabular}


Table 4 Crude and adjusted odds ratio of preterm birth across quintiles of CalEnviroScreen indicators and scores in Fresno County, 2009-2012 ( $N=53,843)$ (Continued)

\begin{tabular}{|c|c|c|c|c|}
\hline Type of Preterm Birth & $<37$ weeks $(N=4560)$ & $\geq 37$ weeks $(N=49,283)$ & & \\
\hline Environmental exposure & $N(\%)$ & $N(\%)$ & COR $(95 \% \mathrm{Cl})$ & $\mathrm{aOR}^{\mathrm{a}}(95 \% \mathrm{Cl})$ \\
\hline \multicolumn{5}{|l|}{ Cleanup Sites } \\
\hline 0 - 19th percentile & $2512(55.1)$ & $26,770(54.3)$ & Reference & Reference \\
\hline 20 - 39th percentile & $537(11.8)$ & $5832(11.8)$ & $0.98(0.90,1.08)$ & $1.01(0.92,1.11)$ \\
\hline 40 - 59th percentile & $561(12.3)$ & $6472(13.1)$ & $0.93(0.85,1.02)$ & $0.90(0.82,0.99)$ \\
\hline 60 - 79th percentile & $486(10.7)$ & $5066(10.3)$ & $1.02(0.93,1.12)$ & $1.01(0.91,1.11)$ \\
\hline 80 - 100th percentile & $464(10.2)$ & $5143(10.4)$ & $0.96(0.87,1.07)$ & $0.94(0.85,1.03)$ \\
\hline \multicolumn{5}{|l|}{ Groundwater Threats } \\
\hline 0 - 19th percentile & $1772(38.9)$ & $19,140(38.8)$ & Reference & Reference \\
\hline 20 - 39th percentile & $683(15.0)$ & $8462(15.1)$ & $0.99(0.91,1.08)$ & $1.00(0.91,1.09)$ \\
\hline 40 - 59th percentile & $976(21.4)$ & $10,124(20.5)$ & $1.04(0.96,1.12)$ & $1.02(0.95,1.11)$ \\
\hline 60 - 79th percentile & $652(14.3)$ & $7472(15.2)$ & $0.95(0.87,1.04)$ & $0.90(0.82,0.99)$ \\
\hline 80 - 100th percentile & $477(10.5)$ & $5085(10.3)$ & $1.01(0.91,1.12)$ & $0.95(0.86,1.06)$ \\
\hline \multicolumn{5}{|l|}{ Hazardous Waste } \\
\hline 0 - 19th percentile & $2274(49.9)$ & $24,740(50.2)$ & Reference & Reference \\
\hline 20 - 39th percentile & 709 (15.6) & $7424(15.1)$ & $1.04(0.95,1.13)$ & $1.01(0.93,1.10)$ \\
\hline 40 - 59th percentile & $658(14.4)$ & 7179 (14.6) & $1.00(0.91,1.09)$ & $0.98(0.90,1.07)$ \\
\hline 60 - 79th percentile & $446(9.8)$ & $5020(10.2)$ & $0.97(0.8,1.07)$ & $0.95(0.86,1.05)$ \\
\hline 80 - 100th percentile & $473(10.4)$ & $4920(10.0)$ & $1.04(0.94,1.15)$ & $1.01(0.92,1.12)$ \\
\hline \multicolumn{5}{|l|}{ Imperial Water Bodies } \\
\hline 0 - 19th percentile & 4031 (88.4) & $42,996(87.2)$ & Reference & Reference \\
\hline 20 - 39th percentile & $286(6.3)$ & $3410(6.9)$ & $0.90(0.80,1.02)$ & $0.90(0.80,1.02)$ \\
\hline 40 - 59th percentile & $162(3.6)$ & $2011(4.1)$ & $0.87(0.74,1.02)$ & $0.84(0.72,0.98)$ \\
\hline 60 - 79th percentile & $38(0.8)$ & $434(0.9)$ & $0.94(0.68,1.29)$ & $0.88(0.64,1.21)$ \\
\hline 80 - 100th percentile & $43(0.9)$ & $432(0.9)$ & $1.06(0.78,1.43)$ & $0.92(0.68,1.24)$ \\
\hline \multicolumn{5}{|l|}{ Solid Waste } \\
\hline 0 - 19th percentile & $2858(62.7)$ & $31,070(63.0)$ & Reference & Reference \\
\hline 20 - 39th percentile & $300(6.6)$ & $3482(7.1)$ & $0.94(0.84,1.06)$ & $0.90(0.79,1.01)$ \\
\hline 40 - 59th percentile & $351(7.7)$ & $3939(8.0)$ & $0.97(0.87,1.09)$ & $0.95(0.85,1.06)$ \\
\hline 60 - 79th percentile & $689(15.1)$ & 7190 (14.6) & $1.04(0.96,1.13)$ & $1.01(0.93,1.10)$ \\
\hline 80 - 100th percentile & $362(7.9)$ & $3602(7.3)$ & $1.08(0.97,1.21)$ & $1.05(0.94,1.17)$ \\
\hline \multicolumn{5}{|l|}{ Pollution Burden Score } \\
\hline 0 - 19th percentile & $17(0.4)$ & $380(0.8)$ & Reference & Reference \\
\hline 20 - 39th percentile & $64(1.4)$ & $1025(2.1)$ & $1.37(0.80,2.34)$ & $1.38(0.79,2.40)$ \\
\hline 40 - 59th percentile & $399(8.8)$ & 4929 (10.0) & $1.75(1.07,2.84)$ & $1.78(1.09,2.88)$ \\
\hline 60 - 79th percentile & $1780(39.0)$ & $18,838(38.2)$ & $2.02(1.25,3.25)$ & $1.98(1.23,3.19)$ \\
\hline 80 - 100th percentile & $2288(50.2)$ & $23,989(48.7)$ & $2.03(1.26,3.28)$ & $1.98(1.23,3.19)$ \\
\hline
\end{tabular}

cOR crude odds ratio, $a O R$ adjusted odds ratio

${ }^{a}$ Adjusted for maternal race/ethnicity, age, education, payment for delivery ${ }^{\dagger} n<16$

NC not calculated (owing to lack of variability)

birth $(<37$ weeks), compared to the lowest quintile in the a priori variable adjustment regardless of different statistical adjustment settings (crude and stepwise adjustment, not shown). We also found the highest three quintiles of Pollution Burden score had statistically higher odds of preterm birth (Table 4).

We found the highest quintile of drinking water contaminants was associated with higher odds of preterm 
Table 5 Crude and adjusted* odds ratio of preterm birth comparing above versus below the median of environmental exposure stratified by census tract-level socioeconomic status (SES) in Fresno County, 2009-2012 (N=53,843)

\begin{tabular}{|c|c|c|c|c|c|c|c|c|}
\hline \multirow{3}{*}{$\begin{array}{l}\text { Environmental } \\
\text { Exposure }\end{array}$} & \multicolumn{4}{|l|}{ LOW SES } & \multicolumn{4}{|l|}{ High SES } \\
\hline & \multirow{2}{*}{$\begin{array}{l}<37 \text { weeks } \\
N(\%)\end{array}$} & \multicolumn{3}{|l|}{$\geq 37$ weeks } & \multirow{2}{*}{$\begin{array}{l}<37 \text { weeks } \\
N(\%)\end{array}$} & \multicolumn{3}{|l|}{$\geq 37$ weeks } \\
\hline & & N (\%) & cOR (95\% Cl) & $\mathrm{aOR}^{*}(95 \% \mathrm{Cl})$ & & $N(\%)$ & cOR (95\% Cl) & aOR* $(95 \% \mathrm{Cl})$ \\
\hline Sample & 2455 & 24,998 & & & 2105 & 24,285 & & \\
\hline \multicolumn{9}{|l|}{ Exposures Score } \\
\hline$<50$ th & 924 (37.6) & $10,360(41.4)$ & Reference & Reference & $1172(55.7)$ & $14,101(58.1)$ & Reference & Reference \\
\hline$\geq 50$ th & 1531 (62.4) & $14,638(58.6)$ & $1.16(1.07,1.25)$ & $1.16(1.06,1.25)$ & $933(44.3)$ & $10,184(41.9)$ & $1.09(1.00,1.19)$ & $1.07(0.98,1.17)$ \\
\hline \multicolumn{9}{|l|}{ Ozone } \\
\hline$<50$ th & $1481(60.4)$ & $15,063(60.3)$ & Reference & Reference & 777 (36.9) & 8752 (36.0) & Reference & Reference \\
\hline$\geq 50$ th & $974(39.7)$ & 9935 (39.7) & $1.00(0.92,1.08)$ & $1.00(0.92,1.08)$ & 1295 (61.5) & $14,916(61.4)$ & $0.98(0.90,1.07)$ & $0.99(0.90,1.08)$ \\
\hline \multicolumn{9}{|l|}{ Pesticides } \\
\hline$<50$ th & 1019 (41.5) & 9872 (39.5) & Reference & Reference & $1244(59.1)$ & $14,691(60.5)$ & Reference & Reference \\
\hline$\geq 50$ th & $1436(58.5)$ & $15,126(60.5)$ & $0.93(0.86,1.00)$ & $0.92(0.85,1.00)$ & 861 (40.9) & 9594 (39.5) & $1.05(0.97,1.15)$ & $1.05(0.97,1.15)$ \\
\hline \multicolumn{9}{|l|}{$\mathrm{PM}_{2.5}$} \\
\hline$<50$ th & $592(24.1)$ & $6434(25.7)$ & Reference & Reference & $1425(67.7)$ & $17,259(71.1)$ & Reference & Reference \\
\hline$\geq 50$ th & 1736 (70.7) & $17,286(69.2)$ & $1.08(0.99,1.19)$ & $1.07(0.98,1.18)$ & $650(30.9)$ & $6492(26.7)$ & $1.19(1.09,1.31)$ & $1.15(1.05,1.26)$ \\
\hline \multicolumn{9}{|l|}{ Diesel PM } \\
\hline$<50$ th & 1096 (44.6) & $12,166(48.7)$ & Reference & Reference & $1051(49.9)$ & $12,587(51.8)$ & Reference & Reference \\
\hline$\geq 50$ th & 1359 (55.4) & $12,832(51.3)$ & $1.16(1.07,1.25)$ & $1.16(1.07,1.25)$ & $1054(50.1)$ & $11,698(48.2)$ & $1.07(0.98,1.17)$ & $1.04(0.96,1.14)$ \\
\hline \multicolumn{9}{|l|}{ Toxic Release } \\
\hline$<50$ th & 735 (29.9) & 8106 (32.4) & Reference & Reference & 1315 (62.5) & $16,391(67.5)$ & Reference & Reference \\
\hline$\geq 50$ th & $1720(70.1)$ & $16,892(67.6)$ & $1.11(1.02,1.21)$ & $1.11(1.02,1.22)$ & $790(37.5)$ & 7894 (32.5) & $1.22(1.12,1.34)$ & $1.18(1.08,1.29)$ \\
\hline \multicolumn{9}{|l|}{ Traffic } \\
\hline$<50$ th & $1240(50.5)$ & $13,288(52.9)$ & Reference & Reference & $972(46.2)$ & $11,579(47.7)$ & Reference & Reference \\
\hline$\geq 50$ th & 1215 (49.5) & $11,770(47.1)$ & $1.09(1.01,1.18)$ & $1.09(1.01,1.18)$ & 1133 (53.8) & $12,706(52.3)$ & $1.05(0.97,1.15)$ & $1.03(0.95,1.13)$ \\
\hline \multicolumn{9}{|l|}{ Drinking Water } \\
\hline$<50$ th & $143(5.8)$ & $1863(7.5)$ & Reference & Reference & $402(19.1)$ & 4713 (19.4) & Reference & Reference \\
\hline$\geq 50$ th & 2312 (94.2) & 23,135 (92.6) & $1.27(1.08,1.51)$ & $1.29(1.09,1.52)$ & $1703(80.90$ & 19,572 (80.6) & $1.02(0.91,1.14)$ & $1.00(0.90,1.12)$ \\
\hline \multicolumn{9}{|l|}{ Arsenic } \\
\hline$<50$ th & $234(9.5)$ & $2467(9.9)$ & Reference & Reference & $493(23.4)$ & $6230(25.7)$ & Reference & Reference \\
\hline$\geq 50$ th & 2221 (90.5) & $22,531(90.1)$ & $1.04(0.91,1.19)$ & $1.01(0.88,1.16)$ & 1612 (76.6) & $18,055(74.4)$ & $1.12(1.01,1.24)$ & $1.09(0.99,1.21)$ \\
\hline \multicolumn{9}{|l|}{ Cadmium } \\
\hline$<50$ th & $0(0.0)$ & $0(0.0)$ & Reference & Reference & $0(0.0)$ & $0(0.0)$ & Reference & Reference \\
\hline$\geq 50$ th & $2455(100.0)$ & $24,998(100.0)$ & NC & NC & $2105(100.0)$ & 24,285 (100.0) & NC & $\mathrm{NC}$ \\
\hline \multicolumn{9}{|c|}{ 1,2-Dibromo-3-chloropropane (DBCP) } \\
\hline$<50$ th & $979(39.9)$ & $10,100(40.4)$ & Reference & Reference & $604(28.7)$ & $6924(28.5)$ & Reference & Reference \\
\hline$\geq 50$ th & $1476(60.1)$ & $14,898(59.5)$ & $1.02(0.94,1.11)$ & $1.02(0.94,1.10)$ & $1472(69.9)$ & $16,834(69.3)$ & $1.00(0.91,1.10)$ & $1.00(0.91,1.10)$ \\
\hline \multicolumn{9}{|c|}{ Hexavalent Chromium } \\
\hline$<50$ th & $0(0.0)$ & $0(0.0)$ & Reference & Reference & $0(0.0)$ & $0(0.0)$ & Reference & Reference \\
\hline$\geq 50$ th & $2147(87.5)$ & $21,281(85.1)$ & NC & NC & $2105(100.0)$ & $24,285(100.0)$ & NC & NC \\
\hline \multicolumn{9}{|l|}{ Lead } \\
\hline$<50$ th & $0(0.0)$ & $0(0.0)$ & Reference & Reference & $0(0.0)$ & $0(0.0)$ & Reference & Reference \\
\hline$\geq 50$ th & $2455(100.0)$ & $24,998(100.0)$ & NC & NC & $2105(100.0)$ & $24,285(100.0)$ & NC & NC \\
\hline
\end{tabular}


Table 5 Crude and adjusted* odds ratio of preterm birth comparing above versus below the median of environmental exposure stratified by census tract-level socioeconomic status (SES) in Fresno County, 2009-2012 ( $N=53,843)$ (Continued)

\begin{tabular}{|c|c|c|c|c|c|c|c|c|}
\hline \multirow{3}{*}{$\begin{array}{l}\text { Environmental } \\
\text { Exposure }\end{array}$} & \multicolumn{4}{|l|}{ Low SES } & \multicolumn{4}{|l|}{ High SES } \\
\hline & \multirow{2}{*}{$\begin{array}{l}<37 \text { weeks } \\
N(\%)\end{array}$} & \multicolumn{3}{|l|}{$\geq 37$ weeks } & \multirow{2}{*}{$\begin{array}{l}<37 \text { weeks } \\
N(\%)\end{array}$} & \multicolumn{3}{|l|}{$\geq 37$ weeks } \\
\hline & & N (\%) & cOR $(95 \% \mathrm{Cl})$ & $\mathrm{aOR}^{*}(95 \% \mathrm{Cl})$ & & $N(\%)$ & cOR $(95 \% \mathrm{Cl})$ & $\mathrm{aOR}^{*}(95 \% \mathrm{Cl})$ \\
\hline \multicolumn{9}{|l|}{ Nitrate } \\
\hline$<50$ th & $1019(41.5)$ & $10,330(41.3)$ & Reference & Reference & $1226(58.2)$ & $14,410(59.3)$ & Reference & Reference \\
\hline$\geq 50$ th & $1436(58.5)$ & $14,668(58.7)$ & $0.99(0.92,1.08)$ & $0.99(0.92,1.07)$ & $879(41.8)$ & $9875(40.7)$ & $1.04(0.96,1.14)$ & $1.03(0.94,1.12)$ \\
\hline \multicolumn{9}{|l|}{ Perchlorate } \\
\hline$<50$ th & $0(0.0)$ & $0(0.0)$ & Reference & Reference & $0(0.0)$ & $0(0.0)$ & Reference & Reference \\
\hline$\geq 50$ th & $2455(100.0)$ & $24,998(100.0)$ & NC & NC & $2105(100.0)$ & $24,285(100.0)$ & NC & NC \\
\hline \multicolumn{9}{|c|}{ Trichloroethylene (TCE) } \\
\hline$<50$ th & $1036(42.2)$ & $11,114(44.5)$ & Reference & Reference & $1182(56.2)$ & $13,607(56.0)$ & Reference & Reference \\
\hline$\geq 50$ th & $1419(57.8)$ & $13,884(55.5)$ & $1.09(1.00,1.18)$ & $1.07(1.00,1.17)$ & $923(43.9)$ & $10,678(44.0)$ & $1.00(0.91,1.09)$ & $0.99(0.91,1.08)$ \\
\hline \multicolumn{9}{|c|}{ Trihalomethane (THM) } \\
\hline$<50$ th & $1145(46.6)$ & $11,838(47.4)$ & Reference & Reference & $859(40.8)$ & $9593(39.5)$ & Reference & Reference \\
\hline$\geq 50$ th & $1310(53.4)$ & $13,160(52.6)$ & $1.03(0.95,1.11)$ & $1.01(0.93,1.09)$ & $1246(59.2)$ & $14,692(60.5)$ & $0.95(0.87,1.04)$ & $0.96(0.88,1.04)$ \\
\hline \multicolumn{9}{|l|}{ Uranium } \\
\hline$<50$ th & $487(19.8)$ & $5535(22.1)$ & Reference & Reference & $294(14.0)$ & $3801(15.7)$ & Reference & Reference \\
\hline$\geq 50$ th & $1968(80.2)$ & $19,463(77.9)$ & $1.15(1.04,1.27)$ & $1.14(1.03,1.25)$ & $1679(79.8)$ & $18,9488(78.0)$ & $1.13(1.00,1.28)$ & $1.12(0.99,1.27)$ \\
\hline \multicolumn{9}{|c|}{ Maximum Contaminant Level (MCL)Violations } \\
\hline$<50$ th & $1030(42.0)$ & $10,577(42.3)$ & Reference & Reference & $1207(57.3)$ & $13,998(57.6)$ & Reference & Reference \\
\hline$\geq 50$ th & $1425(58.0)$ & $14,4211(57.7)$ & $1.01(0.94,1.10)$ & $1.00(0.92,1.09)$ & $898(42.7)$ & $10,287(42.4)$ & $1.01(0.93,1.10)$ & $1.00(0.92,1.09)$ \\
\hline \multicolumn{9}{|c|}{ Total coliform rule (TCR) Violations } \\
\hline$<50$ th & $0(0.0)$ & $0(0.0)$ & Reference & Reference & $0(0.0)$ & $0(0.0)$ & Reference & Reference \\
\hline$\geq 50$ th & $2455(100.0)$ & $24,998(100.0)$ & NC & NC & $2105(100.0)$ & $24,285(100.0)$ & NC & NC \\
\hline \multicolumn{9}{|c|}{ Environmental Effects Score } \\
\hline$<50$ th & $1036(42.2)$ & $10,073(40.3)$ & Reference & Reference & $1254(59.6)$ & $14,381(59.2)$ & Reference & Reference \\
\hline$\geq 50$ th & 1419 (57.8) & $14,925(59.7)$ & $0.93(0.86,1.01)$ & $0.92(0.85,1.00)$ & $851(40.4)$ & $9904(40.8)$ & $0.99(0.90,1.08)$ & $0.98(0.89,1.07)$ \\
\hline \multicolumn{9}{|l|}{ Cleanup Sites } \\
\hline$<50$ th & $1168(47.6)$ & $11,805(47.2)$ & Reference & Reference & $1139(54.1)$ & $12,799(52.7)$ & Reference & Reference \\
\hline$\geq 50$ th & $1287(52.4)$ & $13,193(52.8)$ & $0.99(0.91,1.07)$ & $0.97(0.90,1.05)$ & $966(45.9)$ & $11,486(47.3)$ & $0.95(0.87,1.03)$ & $0.95(0.87,1.04)$ \\
\hline \multicolumn{9}{|c|}{ Groundwater Threats } \\
\hline$<50$ th & $973(39.6)$ & $9545(38.2)$ & Reference & Reference & $1290(61.3)$ & $15,062(62.0)$ & Reference & Reference \\
\hline$\geq 50$ th & $1482(60.4)$ & $15,453(61.8)$ & $0.95(0.87,1.03)$ & $0.93(0.86,1.01)$ & $815(38.7)$ & 9223 (38.) & $1.03(0.94,1.12)$ & $1.02(0.94,1.12)$ \\
\hline \multicolumn{9}{|c|}{ Hazardous Waste } \\
\hline$<50$ th & $1023(41.7)$ & $10,195(40.8)$ & Reference & Reference & $1240(58.9)$ & $14,430(59.4)$ & Reference & Reference \\
\hline$\geq 50$ th & $1432(58.3)$ & $14,803(59.2)$ & $0.97(0.89,1.05)$ & $0.98(0.90,1.06)$ & $865(41.1)$ & 9855 (40.6) & $1.02(0.93,1.11)$ & $0.99(0.91,1.09)$ \\
\hline \multicolumn{9}{|c|}{ Impaired Water Bodies } \\
\hline$<50$ th & $0(0.0)$ & $0(0.0)$ & Reference & Reference & $0(0.0)$ & $0(0.0)$ & Reference & Reference \\
\hline$\geq 50$ th & $2455(100.0)$ & $24,998(100.0)$ & NC & NC & $2105(100.0)$ & $24,285(100.0)$ & NC & NC \\
\hline \multicolumn{9}{|l|}{ Solid Waste } \\
\hline$<50$ th & $0(0.0)$ & $0(0.0)$ & Reference & Reference & $0(0.0)$ & $0(0.0)$ & Reference & Reference \\
\hline$\geq 50$ th & $2455(100.0)$ & $24,998(100.0)$ & NC & NC & $2105(100.0)$ & $24,285(100.0)$ & NC & NC \\
\hline
\end{tabular}


Table 5 Crude and adjusted* odds ratio of preterm birth comparing above versus below the median of environmental exposure stratified by census tract-level socioeconomic status (SES) in Fresno County, 2009-2012 ( $N=53,843)$ (Continued)

\begin{tabular}{|c|c|c|c|c|c|c|c|c|}
\hline \multirow{3}{*}{$\begin{array}{l}\text { Environmental } \\
\text { Exposure }\end{array}$} & \multicolumn{4}{|l|}{ Low SES } & \multicolumn{4}{|l|}{ High SES } \\
\hline & $<37$ weeks & $\geq 37$ weeks & & & $<37$ weeks & $\geq 37$ weeks & & \\
\hline & N (\%) & N (\%) & cOR $(95 \% \mathrm{Cl})$ & $\mathrm{aOR}^{*}(95 \% \mathrm{Cl})$ & $N(\%)$ & $N(\%)$ & cOR $(95 \% \mathrm{Cl})$ & $\mathrm{aOR}^{*}(95 \% \mathrm{Cl})$ \\
\hline \multicolumn{9}{|c|}{ Pollution Burden Score } \\
\hline$<50$ th & $827(33.7)$ & 8615 (35.5) & Reference & Reference & $1380(65.6)$ & $16,068(66.2)$ & Reference & Reference \\
\hline$\geq$ 50th & $1628(66.3)$ & $16,383(65.5)$ & $1.03(0.95,1.12)$ & $1.04(0.96,1.13)$ & $725(34.4)$ & 8217 (33.8) & $1.03(0.94,1.12)$ & $1.03(0.93,1.11)$ \\
\hline
\end{tabular}

NC Not Calculated, cOR crude odds ratio, $a O R$ adjusted odds ratio

*Adjusted for maternal race/ethnicity, age, education, payment for delivery

SES defined as "Socioeconomic Factors" score from the CalEnviroScreen, which includes the following variables derived from the US Census American Community

Survey: educational attainment, linguistic isolation (households where no one over 14 years of age speaks English very well), poverty and unemployment

birth (Table 4), especially spontaneous preterm birth (data not shown). Specifically, uranium concentrations in drinking water was associated with preterm birth and trichloroethylene (TCE) was associated with early preterm birth. Trihalomethanes (THM) concentrations were inversely associated with preterm birth.

The Exposures score, diesel PM and drinking water contaminants were more strongly associated with increased risk of early preterm birth in the low socioeconomic areas compared to the high socioeconomic areas (Table 5). Similar increases were also observed for early preterm birth among the low SES areas compared to high SES areas (Additional file 1: Appendix 3).

We also found the association between diesel PM and preterm birth was slightly higher among non-white and non-Hispanic women, particularly for early preterm birth after adjusting for age, education and payment for delivery costs (Table 6).

\section{Sensitivity analyses}

In logistic regression models of preterm birth ( $<37$ weeks gestation) examining one indicator at a time continuously, two pollutant measures were statistically associated with preterm birth: interquartile range increases in $\mathrm{PM}_{2.5}$ and Pollution Burden Score were associated with $6 \%$ increases in odds of preterm birth after adjustment for education, payer of delivery, maternal age and race/ ethnicity. Diesel PM, traffic density and Trichloroethylene concentration (in drinking water) were associated with $26.3 \%$ increased odds of early preterm birth $(26 \%$, $10 \%, 16 \%$, respectively, Additional file 1: Appendix 2). The associations were consistent between toxic releases and preterm across all race/ethnicity groups, but highest for white, non-Hispanic early preterm births (Additional file 1: Appendix 4). Pesticides were found to be inversely associated with early preterm birth (data not shown).

When all individual environmental indicators and social factors were included in the same model, $\mathrm{PM}_{2.5}$ and unemployment, maternal age $>34$, Medi-Cal payer of delivery and African-American race were associated with preterm birth (data not shown). Results examining raw scores were comparable to those of the percentiles.

\section{Discussion}

Overall, the current study found small but consistent associations between pollution exposure and preterm birth in Fresno County. Although many of the individual pollutants were not associated with preterm birth, the cumulative scores were consistently associated with preterm birth, including the Exposures score, drinking water contaminants and Pollution Burden score. Novel exposures, such as the toxic releases from facilities, were identified as a potential contributor to preterm birth in Fresno County. There was an exposure-response of increased risk of preterm birth across quintiles of Pollution Burden scores. Furthermore, the relationship between pollution and preterm birth was stronger among areas with lower SES.

Some risk factors of preterm birth, such as hypertension, have large associations though only affect a small portion of the population. The associations found with pollution were smaller, but may affect a larger portion of births across the population. Pollution may be exacerbating diseases and health issues that lead to preterm birth (e.g., hypertension) [34], or operating directly through toxic exposures (through a variety of possible mechanisms) [35].

The results did not differ considerably when restricted to spontaneous preterm birth. In some cases, results were stronger among the more severe early preterm birth (less than 34 weeks). The drinking water contaminant, THM, was associated with a decrease in preterm birth; however, it can be inversely correlated with other contaminants because it is a disinfection by-product commonly found in metropolitan areas.

Our findings add to the literature on environmental risk factors and preterm birth. For example, in previous studies in CA, we found small but consistent effects of air pollution on risk of preterm birth using air pollution measurements at the geocoded residence [26, 36, 37]. Along with the current study, two additional studies found 


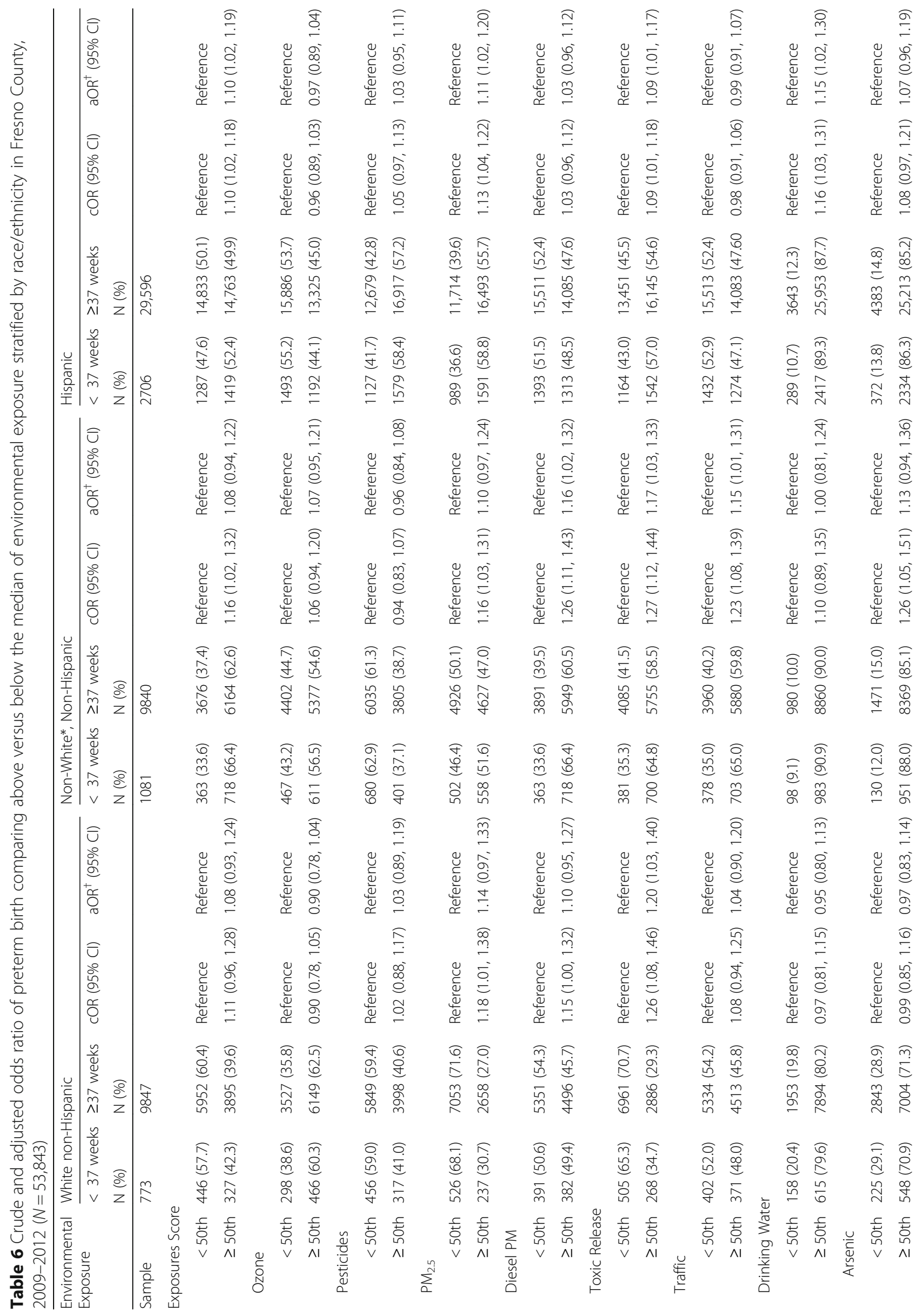




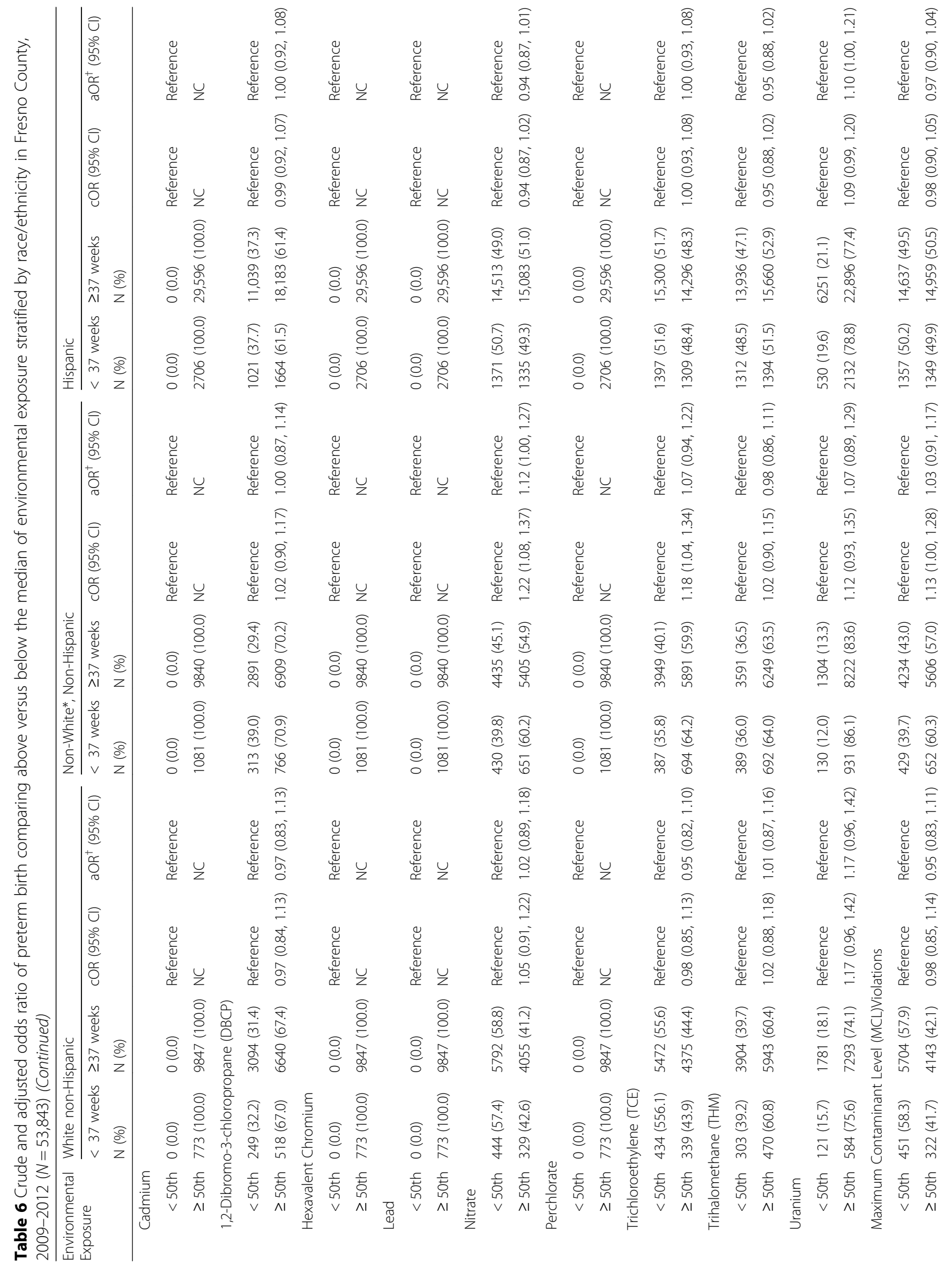




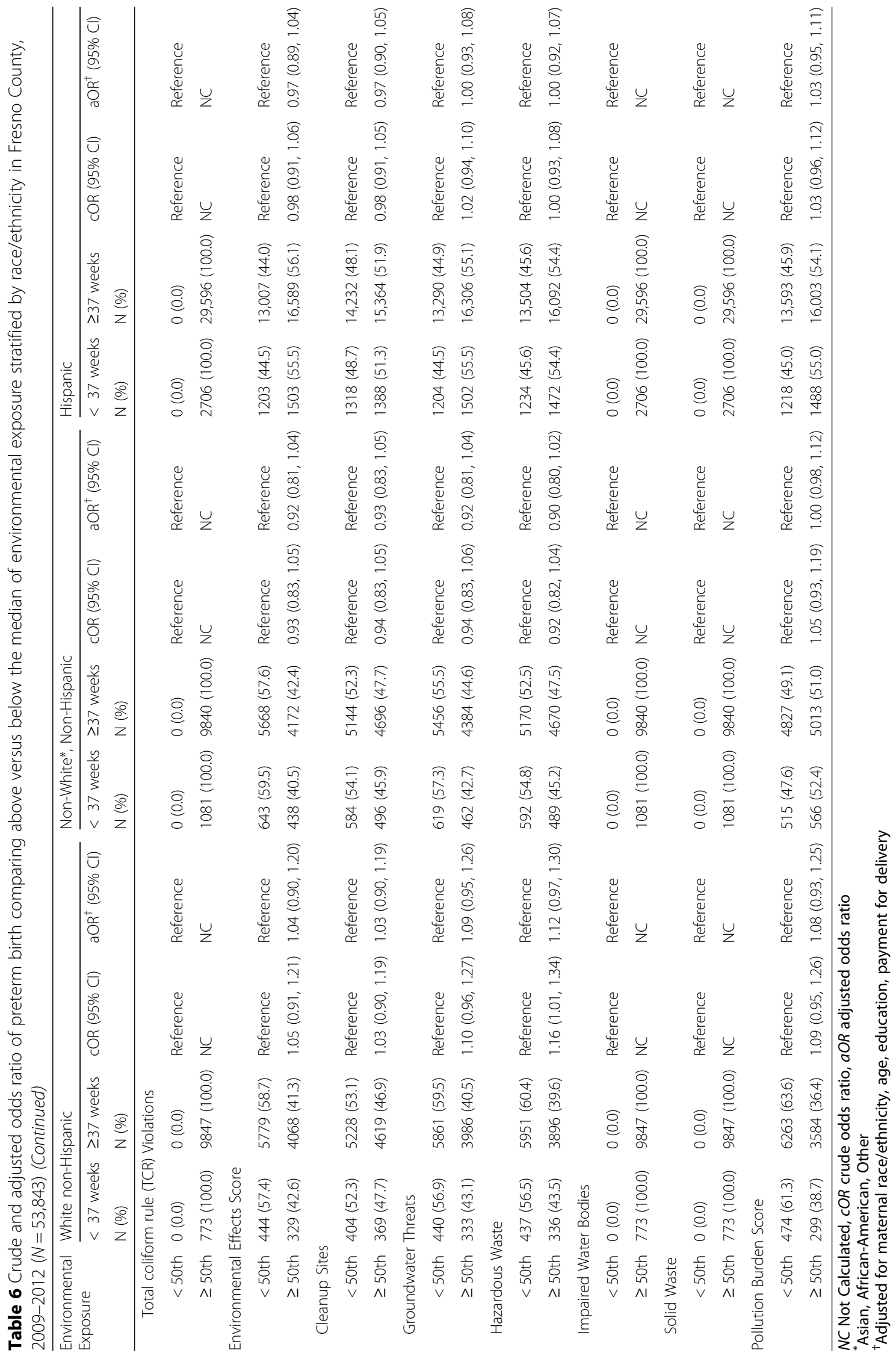


stronger associations between air pollutants and preterm birth for early preterm birth [26,37]. Additionally, an interaction was also observed between air pollution and neighborhood SES using three U.S. Census indicators at the block group level (unemployment, poverty, income from public assistance) [26] in our previous study in the Central Valley of California. Compared to previous studies of air pollution with more precise exposure assessment, our current results are likely underestimated owing to non-differential exposure misclassification. The trade-off of the potential measurement errors is the ability to combine multiple exposures and examine cumulative pollution effects.

Consistent with previous work on environmental justice, we observed higher pollution burden among those who were non-White and of lower education and income. Additionally, we found stronger, though not statistically different, associations between some environmental indicators and preterm birth in low SES areas. This is consistent with the concept of 'double jeopardy' of environmental and socioeconomic stressors [24]. Further work in this area comparing the entire state of California may be more suitable to demonstrate this occurrence. Overall, there were not considerable differences in the association between pollution and preterm birth between racial/ethnic groups.

Notably, WIC participation, which was associated with high pollution burden and requires low SES, was protective against preterm birth. This is an example of a program that may be having a positive effect on reducing preterm birth in Fresno county. The addition of similar programs, which provide access to supplemental foods, healthcare referrals and nutritional education for pregnant women, may further reduce preterm birth in low-income areas.

Despite the large inclusion of the population, our study did have several limitations. One limitation is the imprecise exposure assessment both geographically and temporally. In some cases, the linkage between the birth records and the census tract were not available and this may have resulted in bias, given changes in census tracts are often a result of population growth. The exposure assessment was at the census tract level and the years were pooled for most data sources. Additionally, the CalEnviroScreen was designed as a screening level tool and does not include specific pollutants or chemical exposures that may be affecting this study population. We examined many indicators of pollution that included nested summary measures, which led to many comparisons. Although we did not adjust for multiple comparisons, we present these results as exploratory. Some women may have had two or possibly more births during this time period (2009-2012); however, we were unable to link them and control for these correlated events. Lastly, we assumed that mothers lived constantly throughout their pregnancy in the maternal residence recorded in the birth certificate without relocating from other regions and did not account for time activity patterns or time spent in other geographical areas.

The CalEnviroScreen is a unique tool devised to identify areas of high pollution burden and vulnerable populations and has the benefit of informing epidemiologic studies. Strengths of this study include our ability to include a large set of pollution indicators both individually and cumulatively across a broad geographic area. Additionally, we were able to include all singleton births in Fresno County with detailed demographic and medical information from medical discharge records. Further, our results find a stronger association with the Exposures score, which makes sense as this score consists of monitoring data that is likely to be more representative of actual exposures in the population.

\section{Conclusion}

Our study provides an initial investigation of the CalEnviroScreen as an epidemiologic tool to help elucidate a host of environmental and social factors that contribute to preterm birth. As a screening tool designed to discern communities that assume disproportionate environmental burdens in California, the CalEnviroScreen provides data for environmental justice research. Future studies could expand to the entire state of California and aim to include additional sources of data such as biomonitoring and genomics that could confirm exposure levels and identify pathways by which environmental pollutants contribute to preterm birth.

\section{Additional file}

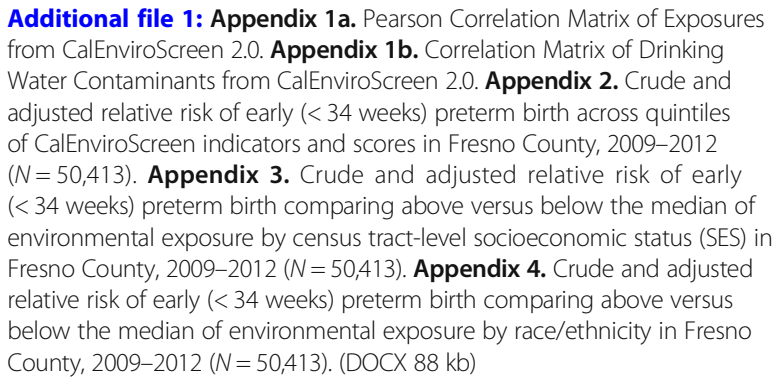

Additional file 1: Appendix 1a. Pearson Correlation Matrix of Exposures from CalEnviroScreen 2.0. Appendix 1b. Correlation Matrix of Drinking Water Contaminants from CalEnviroScreen 2.0. Appendix 2. Crude and adjusted relative risk of early ( $<34$ weeks) preterm birth across quintiles of CalEnviroScreen indicators and scores in Fresno County, 2009-2012 $(N=50,413)$. Appendix 3 . Crude and adjusted relative risk of early ( $<34$ weeks) preterm birth comparing above versus below the median of environmental exposure by census tract-level socioeconomic status (SES) in Fresno County, 2009-2012 ( $N=50,413)$. Appendix 4. Crude and adjusted relative risk of early ( $<34$ weeks) preterm birth comparing above versus below the median of environmental exposure by race/ethnicity in Fresno County, 2009-2012 ( $N=50,413)$. (DOCX $88 \mathrm{~kb})$

\section{Abbreviations}

BMI: Body mass index; CA: California; OSHPD: Office of Statewide Health Planning and Development; PM $_{2.5}$ : Particulate matter $<2.5 \mu \mathrm{m}$ in aerodynamic diameter; SES: Socioeconomic status

\section{Funding}

This research was supported by funding from Mark and Lynn Benioff Preterm Birth Initiative (UCSF7027075) and the National Institutes of Health (NIEHS R00ES021470, NLM K01LM012381). 


\section{Availability of data and materials}

Data for the CalEnviroScreen are publicly available online from the Office of Environmental Health Hazard Assessment of the State of California (https://oehha.ca.gov/calenviroscreen).

Data on the Birth Cohort File are not publicly available but were obtained from the Office of Statewide Health Planning and Development (https://www.oshpd.ca.gov).

\section{Authors' contributions}

AP was the primary author of the manuscript. $\mathrm{HH}$ and RB analyzed the merged CalEnviroScreen and Birth Cohort File. LA provided additional data on water contaminants and insight into interpretation of the environmental data. MJ, LJ, MS, TW were major contributors in writing the manuscript. All authors read and approved the final manuscript.

\section{Ethics approval and consent to participate}

Methods and protocols for the study were approved by the Committee for the Protection of Human Subjects within the Health and Human Services Agency of the State of California.

\section{Consent for publication}

Not applicable

\section{Competing interests}

The authors declare that they have no competing interests.

\section{Publisher's Note}

Springer Nature remains neutral with regard to jurisdictional claims in published maps and institutional affiliations.

\section{Author details}

'Department of Obstetrics, Gynecology and Reproductive Sciences, University of California, 550 16th Street, Mail Stop 0132, San Francisco, CA 94143, USA. ${ }^{2}$ Department of Pediatrics, University of California, San Francisco, USA. ${ }^{3}$ Department of Pediatrics, University of California, San Diego, USA. ${ }^{4}$ Office of Environmental Health Hazard Assessment, California Environmental Protection Agency, Sacramento, USA. ${ }^{5}$ Calit2/Qualcomm Institute, University of California, San Diego, USA. ${ }^{6}$ Department of Epidemiology and Biostatistics, University of California, San Francisco, USA.

\section{Received: 8 December 2017 Accepted: 21 August 2018}

Published online: 29 August 2018

\section{References}

1. McCormick MC, Litt JS, Smith VC, Zupancic JA. Prematurity: an overview and public health implications. Annu Rev Public Health. 2011;32:367-79.

2. Marlow N, Wolke D, Bracewell MA, Samara M. Neurologic and developmental disability at six years of age after extremely preterm birth. N Engl J Med. 2005;352(1):9-19.

3. Anderson P, Doyle LW, Group VICS. Neurobehavioral outcomes of schoolage children born extremely low birth weight or very preterm in the 1990s. JAMA. 2003;289(24):3264-72.

4. Hamilton B, Martin J, Osterman M, et al.: Births: final data for 2014. National vital statistics reports. Hyattsville: National Center for Health Statistics 2015; 64(12).

5. Behrman R, Butler A. Institute of Medicine (US). Committee on understanding premature birth and assuring healthy outcomes. Preterm birth: causes, consequences, and prevention. Washington, DC: National Academies Press; 2007.

6. Sram RJ, Binkova B, Dejmek J, Bobak M. Ambient air pollution and pregnancy outcomes: a review of the literature. Environ Health Perspect. 2005;113(4):375-82.

7. Stieb DM, Chen L, Eshoul M, Judek S. Ambient air pollution, birth weight and preterm birth: a systematic review and meta-analysis. Environ Res. 2012; 117:100-11.

8. Albouy-Llaty M, Limousi F, Carles C, Dupuis A, Rabouan S, Migeot V. Association between exposure to endocrine disruptors in drinking water and preterm birth, taking neighborhood deprivation into account: a historic cohort study. Int J Environ Res Public Health. 2016;13(8) https://doi.org/10. 3390/ijerph13080796.
9. Darrow LA, Stein CR, Steenland K. Serum perfluorooctanoic acid and perfluorooctane sulfonate concentrations in relation to birth outcomes in the mid-Ohio Valley, 2005-2010. Environ Health Perspect. 2013;121(10):1207-13.

10. Kogevinas M, Bustamante M, Gracia-Lavedan E, Ballester F, Cordier S, Costet N, Espinosa A, Grazuleviciene R, Danileviciute A, Ibarluzea J, et al. Drinking water disinfection by-products, genetic polymorphisms, and birth outcomes in a European mother-child cohort study. Epidemiology. 2016;27(6):903-11.

11. Ruckart PZ, Bove FJ, Maslia M. Evaluation of contaminated drinking water and preterm birth, small for gestational age, and birth weight at marine Corps Base camp Lejeune, North Carolina: a cross-sectional study. Environ Health. 2014;13:99.

12. Laine JE, Bailey KA, Rubio-Andrade M, Olshan AF, Smeester L, Drobna Z, Herring AH, Styblo M, Garcia-Vargas GG, Fry RC. Maternal arsenic exposure, arsenic methylation efficiency, and birth outcomes in the biomarkers of exposure to ARsenic (BEAR) pregnancy cohort in Mexico. Environ Health Perspect. 2015;123(2):186-92.

13. Mustafa M, Garg N, Banerjee BD, Sharma T, Tyagi V, Dar SA, Guleria K, Ahmad RS, Vaid N, Tripathi AK. Inflammatory-mediated pathway in association with organochlorine pesticides levels in the etiology of idiopathic preterm birth. Reprod Toxicol. 2015;57:111-20.

14. Kadhel P, Monfort C, Costet N, Rouget F, Thome JP, Multigner L, Cordier S. Chlordecone exposure, length of gestation, and risk of preterm birth. Am J Epidemiol. 2014;179(5):536-44.

15. Eskenazi B, Harley K, Bradman A, Weltzien E, Jewell NP, Barr DB, Furlong CE, Holland NT. Association of in utero organophosphate pesticide exposure and fetal growth and length of gestation in an agricultural population. Environ Health Perspect. 2004;112(10):1116-24.

16. Gehring U, Wijga AH, Fischer P, de Jongste JC, Kerkhof M, Koppelman GH, Smit HA, Brunekreef B. Traffic-related air pollution, preterm birth and term birth weight in the PIAMA birth cohort study. Environ Res. 2011;111(1):125-35.

17. Wilhelm M, Ghosh JK, Su J, Cockburn M, Jerrett M, Ritz B. Traffic-related air toxics and preterm birth: a population-based case-control study in Los Angeles County, California. Environ Health. 2011;10:89.

18. Vafeiadi M, Vriijheid M, Fthenou E, Chalkiadaki G, Rantakokko P, Kiviranta H, Kyrtopoulos SA, Chatzi L, Kogevinas M. Persistent organic pollutants exposure during pregnancy, maternal gestational weight gain, and birth outcomes in the mother-child cohort in Crete, Greece (RHEA study). Environ Int. 2014;64:116-23.

19. Larson K, Russ SA, Nelson BB, Olson LM, Halfon N. Cognitive ability at kindergarten entry and socioeconomic status. Pediatrics. 2015;135(2):E440-8.

20. America's Children and the Environment - Health: Neurodevelopmental Disorders [https://www.epa.gov/ace/health-neurodevelopmental-disorders]. Accessed 23 Aug 2018.

21. Evans GW, Kantrowitz E. Socioeconomic status and health: the potential role of environmental risk exposure. Annu Rev Public Health. 2002;23:303-31.

22. Woodruff TJ, Parker JD, Kyle AD, Schoendorf KC. Disparities in exposure to air pollution during pregnancy. Environ Health Perspect. 2003;111(7):942-6.

23. DeFur PL, Evans GW, Hubal EAC, Kyle AD, Morello-Frosch RA, Williams DR. Vulnerability as a function of individual and group resources in cumulative risk assessment. Environ Health Perspect. 2007:115(5):817-24.

24. Morello-Frosch R, Shenassa ED. The environmental "riskscape" and social inequality: implications for explaining maternal and child health disparities. Environ Health Perspect. 2006;114(8):1150-3.

25. Justice LOMCOE: Toward environmental justice: research, education, and health policy needs: National Academies Press (US); 1999.

26. Padula AM, Mortimer KM, Tager IB, Hammond SK, Lurmann FW, Yang W, Stevenson DK, Shaw GM. Traffic-related air pollution and risk of preterm birth in the San Joaquin Valley of California. Ann Epidemiol. 2014;24(12):888-895e884.

27. Place Matters For Health in the San Joaquin Valley. Ensuring Opportunities for Good Health for All. Washington, DC: Joint Center for Political and Economic Studies; 2012.

28. Talge NM, Mudd LM, Sikorskii A, Basso O. United States birth weight reference corrected for implausible gestational age estimates. Pediatrics. 2014;133(5):844-53.

29. California Communities Environmental Health Screening Tool, Version 2.0 (CalEnviroScreen 2.0): Guidance and Screening Tool. [https://oehha.ca.gov/ media/CES20FinalReportUpdateOct2014.pdf]. Accessed 23 Aug 2018.

30. CalEnviroScreen [http://oehha.ca.gov/calenviroscreen/report/ calenviroscreen-version-20]. Accessed 23 Aug 2018.

31. Alexeeff GV, Faust JB, August LM, Milanes C, Randles K, Zeise L, Denton J. A screening method for assessing cumulative impacts. Int J Environ Res Public Health. 2012;9(2):648-59. 
32. Morello-Frosch R, Jesdale BM, Sadd JL, Pastor M. Ambient air pollution exposure and full-term birth weight in California. Environ Health. 2010;9:44.

33. Shachar BZ, Mayo JA, Lyell DJ, Baer RJ, Jeliffe-Pawlowski LL, Stevenson DK, Shaw GM. Interpregnancy interval after live birth or pregnancy termination and estimated risk of preterm birth: a retrospective cohort study. BJOG. 2016;123(12):2009-17.

34. Lavigne E, Yasseen AS 3rd, Stieb DM, Hystad P, van Donkelaar A, Martin RV, Brook JR, Crouse DL, Burnett RT, Chen H, et al. Ambient air pollution and adverse birth outcomes: differences by maternal comorbidities. Environ Res. 2016;148:457-66.

35. Ferguson KK, Chin HB. Environmental chemicals and preterm birth: biological mechanisms and the state of the science. Curr Epidemiol Rep. 2017:4(1):56-71.

36. Huynh M, Woodruff TJ, Parker JD, Schoendorf KC. Relationships between air pollution and preterm birth in California. Paediatr Perinat Epidemiol. 2006; 20(6):454-61.

37. Padula AM, Noth EM, Hammond SK, Lurmann FW, Yang W, Tager IB, Shaw GM. Exposure to airborne polycyclic aromatic hydrocarbons during pregnancy and risk of preterm birth. Environ Res. 2014;135:221-6.

Ready to submit your research? Choose BMC and benefit from:

- fast, convenient online submission

- thorough peer review by experienced researchers in your field

- rapid publication on acceptance

- support for research data, including large and complex data types

- gold Open Access which fosters wider collaboration and increased citations

- maximum visibility for your research: over $100 \mathrm{M}$ website views per year

At $\mathrm{BMC}$, research is always in progress.

Learn more biomedcentral.com/submissions 